\title{
Split Membrane 11D Spacetime = 1D Eleventh Dimension Interval Space + 6D Rishon Space + 3D Higgs Space + 1D Einstein Time: Cosmology
}

\author{
Ding-Yu Chung \\ Utica, Michigan, USA \\ Email:dy_chung@yahoo.com
}

How to cite this paper: Chung, D.-Y. (2019) Split Membrane 11D Spacetime = 1D Eleventh Dimension Interval Space + $6 \mathrm{D}$ Rishon Space $+3 \mathrm{D}$ Higgs Space $+1 \mathrm{D}$ Einstein Time: Cosmology. Journal of Modern Physics, 10, 1310-1341. https://doi.org/10.4236/jmp.2019.1011087

Received: August 30, 2019

Accepted: October 9, 2019

Published: October 12, 2019

Copyright ( 2019 by author(s) and Scientific Research Publishing Inc. This work is licensed under the Creative Commons Attribution International License (CC BY 4.0).

http://creativecommons.org/licenses/by/4.0/

\begin{abstract}
The paper posits that the cyclic universe cosmology involves the split of the membrane 11D (11 dimensional) spacetime into the 1D eleventh dimension orbifold interval space to form gravity, the $6 \mathrm{D}$ discrete interior rishon space (TTT-VVV for positron-neutrino or TTV-TVV for u-d quarks) to form the Standard Model, the 3D Higgs space (attachment space to attach matter or detachment space to detach matter) to form the Higgs or reverse Higgs field, and 1D Einstein time to be shared by all spaces. To establish particle masses, spacetime dimension number decreases with decreasing speed of light, decreasing vacuum energy, and increasing rest mass. The $4 \mathrm{D}$ and the $10 \mathrm{D}$ have zero and the highest vacuum energies, respectively. The cyclic universe cosmology starts with the zero-energy $4 \mathrm{D}$ inter-universal void and the positive-energy membrane and negative-energy antimembrane 11D dual universe which is split into four equal $10 \mathrm{D}$ string branes, including the $10 \mathrm{D}$ positive-energy weak-gravity brane with matter, negative-energy strong-gravity brane, negative-energy weak-gravity brane with antimatter, and positive-energy strong-gravity brane in the $11 \mathrm{D}$ bulk with the $1 \mathrm{D}$ eleventh dimension interval space in between the strong and the weak-gravity branes. To form the home universe where we inhabit, the 10D positive-energy weak-gravity brane with attachment space absorbed the zero-energy $4 \mathrm{D}$ inter-universal void with detachment space, resulting in the combination of rest mass from attachment space and kinetic energy from detachment space, the formation of the $4 \mathrm{D}$ spacetime universe by transforming $6 \mathrm{D}$ connected exterior space into $6 \mathrm{D}$ discrete interior rishon space, and cosmic inflation. The other three branes did not absorb the inter-universal void, resulting in the oscillating dimension branes between 10D and 4D stepwise without kinetic energy. The three branes are hidden when $\mathrm{D}>4$, and they are dark energy when $\mathrm{D}=4$. The split $11 \mathrm{D}$ spacetime and cosmology provide the mat-
\end{abstract}


ter-antimatter imbalance and the accurately calculated masses for leptons, quarks, hadrons, gauge bosons, the Higgs boson, gravity, dark matter, and dark energy.

\section{Keywords}

String Theory, Rishon, Cyclic Universe Cosmology, Dark Energy, Dark Matter, Particle Masses

\section{Introduction}

String theory proposes to produce a theory of everything that describes all known particles and forces [1]. String theory with the inclusion of the 11th dimension is called M-theory. The extra dimensions of 11D (11 dimensional) M-theory or $10 \mathrm{D}$ string theory are the compact manifolds with sizes about the order of the Planck length, $10^{-33} \mathrm{~cm}$, such that they remain hidden to the experiment, explaining why we see only four dimensions. The compactification of the extra space dimensions specifies a shape for the extra space dimensions. Different shapes correspond to different possible universes. The number of the shapes is typically estimated to be around $10^{500}$, which can accommodate almost any phenomena [2].

To avoid the problem of $10^{500}$ universes due to the compactification, an alternative to the compactification is the Randall-Sundrum model for five dimensional spacetime [3]. In the RS1 (Randall-Sundrum model 1) of the Randall-Sundrum model [4] [5], the fifth dimension as the extra space dimension is an orbifold interval space between the Planckbrane (strong-gravity brane) and the Tevbrane (weak-gravity brane) in the warped five dimensional bulk. In this warped spacetime which is only warped along the fifth dimension, the gravity is extremely high at the strong-gravity brane, but it drops exponentially as it moves closer towards the weak-gravity brane. Gravity on the weak-gravity brane is much weaker than on the strong-gravity brane. The weak-gravity brane contains matter as the Standard Model particles, while the strong-gravity brane is dominated by gravity. The Randall-Sundrum model explains the hierarchy problem between the weak-gravity and the strong electromagnetic force and the other fundamental forces. Using the Randall-Sundrum model, this paper posits that the $1 \mathrm{D}$ eleventh dimension interval space was the space between the two string branes (the weak-gravity string brane and the strong-gravity string brane) in the warped 11D membrane bulk. The two string 10-branes are the progenitors of the home universe where we inhabit and dark energy. The comparison between the RS1-5D spacetime and the RS1-11D spacetime is as Figure 1.

The Standard Model of particle physics consists of 17 elementary particles including 6 leptons (electron, muon, and tau, electron neutrino, muon neutrino, tau neutrino), 6 quarks (down, up, strange, charm, bottom, and top), 4 gauge bosons (photon, $\mathrm{W}$ boson, $\mathrm{Z}$ boson, and gluon), and the Higgs boson. The 4 


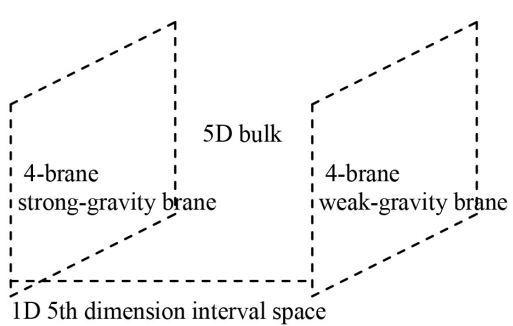

(a) RS1- 5D spacetime

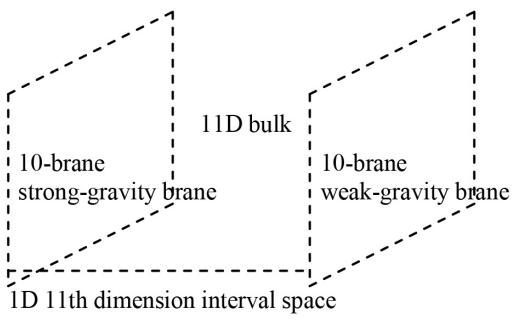

(b) RS1- 11D spacetime

Figure 1. (a) RS1-5D spacetime: the 5D bulk with the two 4-branes (the weak-gravity brane and the strong-gravity brane) and 1D 5th dimension interval space. (b) RS1-11D spacetime: the $11 \mathrm{D}$ bulk with the two 10-branes (the weak-gravity brane and the strong-gravity brane) and 1D 11th dimension interval space.

gauge bosons have the gauge symmetry U(1) X SU(3) X SU(2) . The Standard Model explains very well the relations among the 17 elementary particles, but the Standard Model cannot explain the masses of 17 elementary particles, the origins of the 17 elementary particles and the gauge symmetry, neutrino oscillation, the apparent matter-antimatter imbalance in the universe, gravity, dark matter, and dark energy.

To explain the origin of leptons and quarks in the Standard Model, preon models propose simple substructure particles in leptons and quarks [6] [7]. One of the preon models is the Rishon Model by Haim Harari [8] and Michael A. Shupe [9]. The two fundamental substructure particles are rishons consisting of $\mathrm{T}$ with $1 / 3$ charge and $1 / 2$ spin and neutral $\mathrm{V}$ with $1 / 2$ spin. TTT is positron, while VVV is electron neutrino. TTV, TVT and VTT are the three colors of up quark, while TVV, VTV and VVT are the three colors of down quark. Hypercolor is required to confine rishons in leptons and quarks [10]. The Rishon Model provides the origin of the first generation fermions in the Standard Model, but there is no experimental evidence for the existence of rishons as the substructure particles Quarks and leptons have been found to be point-like particles without substructure particles [11].

To avoid the difficulty of substructure particles of the Rishon model, Sundance Bilson-Thompson proposes the Helon Model with the discrete topological properties of discrete trivalent twisted strand braids [12] [13]. Each rishon is a helon $\left(\mathrm{H}_{+}, \mathrm{H}_{-}\right.$, or $\left.\mathrm{H}_{0}\right)$ which is a twisted strand, and three twisted strands form a braid in the Helon Model. $\mathrm{H}_{+} \mathrm{H}_{+} \mathrm{H}_{+}, \mathrm{H}_{0} \mathrm{H}_{0} \mathrm{H}_{0}, \mathrm{H}_{+} \mathrm{H}_{+} \mathrm{H}_{0}$, and $\mathrm{H}_{+} \mathrm{H}_{0} \mathrm{H}_{0}$ correspond to TTT, VVV, TTV, and TVV, respectively.

This paper proposes the Rishon Space Model where rishon is space instead of substructure particle. The Rishon Space Model is derived from the transformation from 10D string spacetime into $4 \mathrm{D}$ observed spacetime by converting the $6 \mathrm{D}$ connected space into the $6 \mathrm{D}$ discrete rishon space in the form of the two sets of three (trivalent) discrete one-dimensional strands as in the Helon Model. The 6D discrete rishon space consists of TTT-VVV or TTV-TVV space for the space of positron-neutrino or u-d quarks, respectively. The two sets of the trivalent 
dimensional space in the $6 \mathrm{D}$ rishon space have the symmetry of isospin between TTT and VVV and between TTV and TVV. TTT represents one integer charge, VVV is neutral, and TTV and TVV represent fractional charges [14]. The reason for the confined trivalent rishon space is to match the confined trivalent $3 \mathrm{D}$ connected space in the observed $4 \mathrm{D}$ spacetime, so the trivalent discrete rishon space becomes the interior space to determine the properties (flavor, charge, isospin, and color) of leptons and quarks, while the trivalent connected space becomes the exterior space to determines the momenta and positions of leptons and quarks.

The transformation from 10D string spacetime into $4 \mathrm{D}$ observed by converting the 6D connected space into the 6D Rishon Space with TTTVVV or TTVTVV is the continuation of the RS1-11D spacetime where the 10D weak-gravity brane is converted into the $4 \mathrm{D}$ home universe where we inhibit with the $6 \mathrm{D}$ rishon space as Figure 2.

In conventional Higgs mechanism, the Higgs field is the place where a massless particle gains mass through spontaneous symmetry breaking. The scalar Higgs Field exists permanently in the universe. The problem with such permanent Higgs field is the cosmological constant problem from the huge gravitational effect by the Higgs field in contrast to the observation [15]. To avoid the cosmological problem, the previous paper [16] posits that the Higgs field is transitional, and appears only during spontaneous symmetry breaking. The Higgs field disappears after spontaneous symmetry breaking. The permanent space precursor of the Higgs field is attachment space that attaches matter to space, and relates to rest mass. The permanent space precursor of the transitional reverse Higgs field is 3D detachment space which detaches matter from space, and relates to kinetic energy. Attachment space and detachment space are the Higgs space which interacts with matter. Because the Higgs field and the reverse Higgs field are transitional, the cosmological constant problem disappears. The mixed Higgs spaces as the combinations of $\mathrm{n}$ units of attachment space (denoted as 1 ) and $\mathrm{n}$ units of detachment space (denoted as 0 ) consist of binary partition space, $(1) n(0) n$, as the space of wave-particle duality, binary miscible space, $(1+0) n$, as the space of relativity, and binary lattice space, $\left(\begin{array}{ll}1 & 0\end{array}\right) \mathrm{n}$, as the space of virtual gauge rishon boson in quantum field theory. The Higgs space is the interior space to determine the properties (attachment and detachment to matter) of

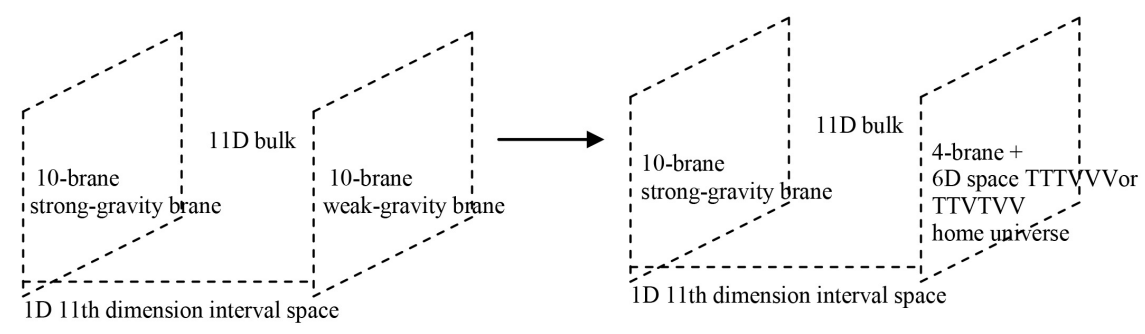

Figure 2. The transformation from the $10 \mathrm{D}$ weak-gravity brane to the home universe with $4 \mathrm{D}$ spacetime $+6 \mathrm{D}$ space (TTTVVV or TTVTVV). 
elementary particles, while the exterior space determines the momenta and positions of elementary particles. A particle with the discrete interior rishon space moves with the different bound Higgs spaces under different conditions.

As shown later, detachment space is originally from the inter-universal void in the multiverse. To form the home universe where we inhabit, the 10D weak-gravity brane with attachment space absorbed the zero-energy $4 \mathrm{D}$ inter-universal void with detachment space, resulting in the combination of rest mass from attachment space and kinetic energy from detachment space and the formation of the $4 \mathrm{D}$ spacetime universe by transforming $6 \mathrm{D}$ connected exterior space into 6D discrete interior rishon space as Figure 3.

According to Johan Hansson, one of the ten biggest unsolved problems (quantum gravity, particle masses, the "measurement" problem, turbulence, dark energy, dark matter, complexity, the matter-antimatter asymmetry, friction, and the "arrow of time") in physics [17] is the incalculable particle masses of leptons, quarks, gauge bosons, and the Higgs boson. The Standard Model of particle physics contains the particles masses of leptons, quarks, and gauge bosons which cannot be calculated or predicted theoretically. From a theoretical point of view the particle mass is a total unsolved problem - they might as well have been random numbers drawn from a hat. The repetition of leptons and quarks with increasing masses has also remained an unsolved problem. Max Jammer [18] concluded that nobody knows what particle masses really are. The mass parameters experimentally measured for elementary particles have no theoretical explanation whatsoever. From the vantage point of theory the masses could just as well be a set of randomly generated numbers.

In this paper, the masses of elementary particles are calculated accurately by dimension numbers. As discussed in the previous papers [19] [20], between 4D spacetime and 10D spacetime, dimension number decreases with decreasing speed of light, decreasing vacuum energy, and increasing rest mass. The 4D and the 10D have zero and the highest vacuum energies, respectively. Each spacetime dimension $\mathrm{D}$ associates with mass dimension $\mathrm{d}$ where $\mathrm{D}+\mathrm{d}=14$ initially. All elementary particles for baryonic matter (leptons, quarks, gauge bosons, gravity, the Higgs boson, and cosmic rays) and dark matter (sterile neutrinos) can be placed in the periodic table of elementary particles based on the two sets of the seven mass dimensions [21] [22]. The periodic table of elementary particles

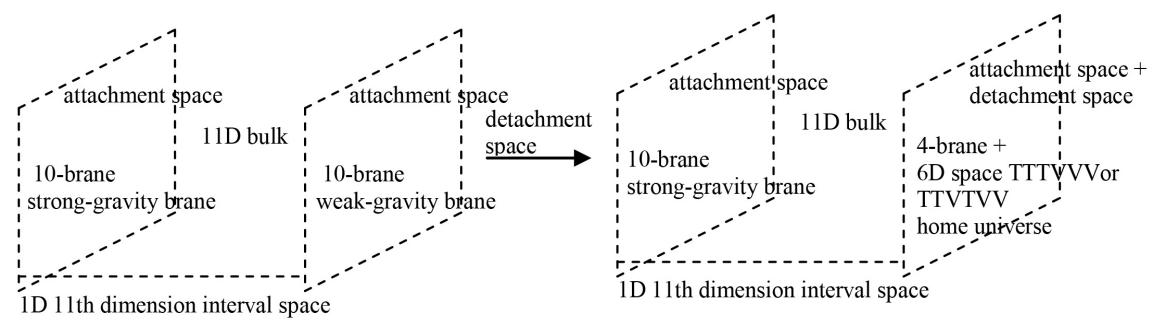

Figure 3. The addition of detachment space from the zero-energy 4D inter-universal void to the $10 \mathrm{D}$ weak-gravity brane to form the home universe with both attachment space and detachment space. 
provide the accurate calculated masses for leptons, quarks, hadrons, gauge bosons, the Higgs boson, gravity, dark matter, and dark energy.

This paper posits that the origin of the split is from the cyclic universe cosmology in the multiverse. As mentioned in the previous papers [19] [20], the multiverse consists of the zero-energy inter-universal void with 4D detachment space and the multiple 11D positive-energy membrane-antimembrane and negative-energy anti-membrane dual universes with $11 \mathrm{D}$ attachment space. The total energy of a dual 11D universe is zero. Detachment space in the inter-universal void prevents the collision of the 11D dual universes, while attachment space in a dual universe allows the existence of mass-energy in the space of the dual universe.

The cyclic universe cosmology starts with the zero-energy inter-universal void and the positive-energy membrane and negative-energy antimembrane 11D dual universe which is split into four equal 10D string branes, including the 10D positive-energy weak-gravity brane with matter, negative-energy strong-gravity brane, negative-energy weak-gravity brane with antimatter, and positive-energy strong-gravity brane in the $11 \mathrm{D}$ bulk. The $1 \mathrm{D}$ eleventh dimension interval space is between the strong and the weak-gravity branes as in Randall-Sundrum model. To form the home universe where we inhabit, the positive-energy weak-gravity brane with attachment space absorbed the zero-energy $4 \mathrm{D}$ inter-universal void with detachment space, resulting in the combination of rest mass from attachment space and kinetic energy from detachment space and the formation of the $4 \mathrm{D}$ spacetime universe by transforming $6 \mathrm{D}$ connected exterior space into the $6 \mathrm{D}$ discrete interior rishon space. The result was cosmic inflation from high vacuum energy (10D) to zero vacuum energy (4D). The other three branes did not absorb the inter-universal void, resulting in the oscillating dimension branes between 10D and 4D stepwise without kinetic energy. The three branes are hidden when $\mathrm{D}>4$, and they are dark energy when $\mathrm{D}=4$. Afterward, when $\mathrm{D}>4$ for the three branes, the home universe and the three branes start to contract, and eventually return to the original four $10 \mathrm{D}$ string branes and then to the original $11 \mathrm{D}$ membrane-antimembrane dual universe for the cyclic universe cosmology. Different spacetimes are shown in Table 1.

Section 2 deals with the rishon space. Section 3 describes the Higgs space. Section 4 explains the spilt membrane 11D spacetime based on the cyclic universe cosmology in the multiverse. The 11D split spacetime and cosmology provides the matter-antimatter imbalance and the accurate calculated masses for all elementary baryonic matter particles, dark matter, and dark energy.

\section{The Rishon Space}

The paper posits that the membrane 11D spacetime is split into the $1 \mathrm{D}$ eleventh dimension orbifold interval space, the $6 \mathrm{D}$ discrete topological rishon space, the 3D bound Higgs space, and the 1D Einstein time. The 6D discrete topological rishon space is derived from the Rishon Model by Haim Harari [8] and Michael A. Shupe [9] which explains the origin of leptons and quarks in the Standard 
Table 1. Spacetimes.

\begin{tabular}{|c|c|c|c|}
\hline spacetime & origin & topology & relate to \\
\hline $11 \mathrm{D}$ spacetime & original 11D membrane universe & large and connected & attachment space for rest mass \\
\hline 10D spacetime & original $10 \mathrm{D}$ string brane & large and connected & attachment space for rest mass \\
\hline $\begin{array}{l}\text { oscillating D } \\
\text { spacetime }\end{array}$ & the three oscillating branes between $10 \mathrm{D}$ and $4 \mathrm{D}$ & large and connected & dark energy when $\mathrm{D}=4$ \\
\hline $\begin{array}{l}\text { 1D } 11 \text { th dimension } \\
\text { interval space }\end{array}$ & $\begin{array}{l}\text { interval space between the two string branes in } 11 \mathrm{D} \\
\text { membrane bulk }\end{array}$ & orbifold interval space & gravity \\
\hline $6 \mathrm{D}$ rishon space & $\begin{array}{l}\text { transformation from } 10 \mathrm{D} \text { spacetime to } 4 \mathrm{D} \text { spacetime by } \\
\text { transforming } 6 \mathrm{D} \text { connected exterior space into } 6 \mathrm{D} \text { discrete } \\
\text { interior rishon space }\end{array}$ & $\begin{array}{l}\text { discrete trivalent } \\
\text { twisted strand braids }\end{array}$ & $\begin{array}{l}\text { space (TTT-VVV or TTV-TVV) of } \\
\text { positron-neutrino or up-down quarks }\end{array}$ \\
\hline 3D Higgs space & $\begin{array}{l}\text { the absorption of } 4 \mathrm{D} \text { inter-universal void by the } 10 \mathrm{D} \\
\text { string brane }\end{array}$ & bound to all spaces & $\begin{array}{l}\text { the space precursors of the transitional } \\
\text { Higgs field and reverse Higgs field }\end{array}$ \\
\hline 1D Einstein time & $11 \mathrm{D}$ spacetime and $4 \mathrm{D}$ spacetime & bound to all spaces & all split spaces share the same time \\
\hline
\end{tabular}

Model. The Rishon Model proposes that the two fundamental substructure particles are rishons consisting of $T$ with $1 / 3$ charge and $1 / 2$ spin and neutral $V$ with $1 / 2$ spin. TTT is positron, while VVV is electron neutrino. TTV, TVT and VTT are the three colors of up quark, while TVV, VTV and VVT are the three colors of down quark. The Rishon Model provides the origin of the first generation fermions in the Standard Model, but there is no experimental evidence for the existence of rishons as the substructure particles. Quarks and leptons have been found to be point-like particles without substructure particles [11].

To avoid the difficulty of substructure particles, Sundance Bilson-Thompson proposes the Helon Model with the discrete topological properties of discrete trivalent twisted strand braids [12] [13]. Each rishon is a helon which is a twisted strand as $\mathrm{H}_{+}, \mathrm{H}_{-}$, or $\mathrm{H}_{0}$ as shown in Figure 4. In the helon model, helons are topological in nature, and helons are not considered as substructure particles. The integral twists of strands represent the quantized electric charges of particles. Three twisted strands form a braid in the Helon Model. $\mathrm{H}_{+} \mathrm{H}_{+} \mathrm{H}_{+}, \mathrm{H}_{0} \mathrm{H}_{0} \mathrm{H}_{0}, \mathrm{H}_{+}$ $\mathrm{H}_{+} \mathrm{H}_{0}$, and $\mathrm{H}_{+} \mathrm{H}_{0} \mathrm{H}_{0}$ as the trivalent twisted strand braids represent positron, neutrino, up quark, and down quark, respectively. The permutations of twists on certain braids naturally account for the color charges of quarks and gluons.

This paper proposes the Rishon Space Model where rishon is space instead of substructure particle. The Rishon Space Model is derived from the transformation from 10D string spacetime into $4 \mathrm{D}$ observed spacetime by converting the $6 \mathrm{D}$ connected space into the $6 \mathrm{D}$ discrete rishon space in the form of the two sets of three (trivalent) discrete one-dimensional strands as in the Helon Model. The 6D discrete rishon space consists of TTT-VVV or TTV-TVV space for the space of positron-neutrino or u-d quarks, respectively. The two sets of the trivalent dimensional space in the $6 \mathrm{D}$ rishon space have the symmetry of isospin between TTT and VVV and between TTV and TVV. TTT represents one integer charge, VVV is neutral, and TTV and TVV represent fractional charges. The reason for the confined trivalent rishon space is to match the confined trivalent $3 \mathrm{D}$ 


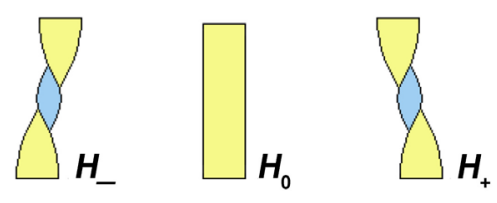

Figure 4. Twist helons.

connected space in the observed $4 \mathrm{D}$ spacetime, so the trivalent discrete rishon space becomes the interior space to determine the properties (flavor, charge, isospin, and color) of leptons and quarks, while the trivalent connected space becomes the exterior space to determines the momenta and positions of leptons and quarks. The transformation from the $6 \mathrm{D}$ connected exterior space into $6 \mathrm{D}$ discrete interior rishon space is as follows.

9D connected exterior space in 10D connected exterior spacetime for string

$\stackrel{\text { from 10D to 4D }}{\longrightarrow} 6 \mathrm{D}$ discrete interior rishon space(TTTVVV) for e- $v$ in $4 \mathrm{D}$

connected exterior spacetime or 6D discrete interior rishon space(TTVTVV) (1)

for $\mathrm{u}-\overline{\mathrm{d}}$ in $4 \mathrm{D}$ connected exterior spacetime $\longrightarrow$ TTT for e and VVV for $v$

or TTV for $\mathrm{u}$ and TVV for $\overline{\mathrm{d}}$ in $4 \mathrm{D}$ connected exterior spacetime

The rishon space and helon are shown in Table 2 and Figure 5. The interactions among leptons and quarks are through the 4 gauge bosons (photon, $\mathrm{W}$ boson, $\mathrm{Z}$ boson, and gluon). The 4 gauge bosons have the gauge symmetry $\mathrm{U}(1)$ $\mathrm{X} \mathrm{SU}(3)_{\mathrm{c}} \mathrm{X} \mathrm{SU}(2)_{\mathrm{L}}$.

\section{The Higgs Space}

The 3D Higgs space consists of attachment space as the space precursor of the transitional Higgs field and detachment space as the space precursor of the transitional reverse Higgs field. Attachment space that attaches matter to the space relates to rest mass, detachment space that detaches matter from the space relates to kinetic energy. The Higgs space involves the Higgs mechanism for the transformation between massless particle and massive particle and the space structures from the combination of attachment space and detachment space. The Higgs space is the interior space to determine the properties (attachment and detachment to matter) of elementary particles, while the exterior space determines the momenta and positions of elementary particles.

\subsection{The Higgs Mechanism}

In conventional physics, space does not couple with particles, and is the passive zero-energy ground state space. Under spontaneous symmetry breaking in conventional physics, the passive zero-energy ground state is converted into the active, permanent, and ubiquitous nonzero-energy Higgs field, which couples with massless particle to produce the transitional Higgs field-particle composite. Under spontaneous symmetry restoring, the transitional Higgs field-particle composite is converted into the massive particle with the longitudinal component on zero-energy ground state without the Higgs field as follows. 
Table 2. The rishon space and helon.

\begin{tabular}{ccc}
\hline particles & rishon space & helon \\
\hline positron & TTT & $\mathrm{H}_{+} \mathrm{H}_{+} \mathrm{H}_{+}$ \\
electron & $\overline{\text { TTT }}$ & $\mathrm{H}_{-} \mathrm{H}_{-} \mathrm{H}_{-}$ \\
neutrino & VVV & $\mathrm{H}_{0} \mathrm{H}_{0} \mathrm{H}_{0}$ \\
u quark & TTV, TVT, VTT & $\mathrm{H}_{+} \mathrm{H}_{+} \mathrm{H}_{0} . \mathrm{H}_{+} \mathrm{H}_{0} \mathrm{H}_{+}, \mathrm{H}_{0} \mathrm{H}_{+} \mathrm{H}_{+}$, \\
$\bar{d}$ quark & TVV, VTV. VTT & $\mathrm{H}_{+} \mathrm{H}_{0} \mathrm{H}_{0}, \mathrm{H}_{0} \mathrm{H}_{+} \mathrm{H}_{0} \mathrm{H}_{0}, \mathrm{H}_{0} \mathrm{H}_{0} \mathrm{H}_{+}$ \\
$\bar{u}$ quark & $\overline{T T V}, \overline{T V T}, \overline{\text { VTT }}$ & $\mathrm{H}_{-} \mathrm{H}_{-} \mathrm{H}_{0}, \mathrm{H}_{-} \mathrm{H}_{0} \mathrm{H}_{-}, \mathrm{H}_{-} \mathrm{H}_{-} \mathrm{H}_{0}$ \\
$d$ quark & $\overline{T V V}, \overline{\text { VTV }}, \overline{\mathrm{V} T \bar{T}}$ & $\mathrm{H}_{-} \mathrm{H}_{0} \mathrm{H}_{0}, \mathrm{H}_{0} \mathrm{H}_{-} \mathrm{H}_{0}, \mathrm{H}_{0} \mathrm{H}_{0} \mathrm{H}_{-}$
\end{tabular}

left handed space

rishon space

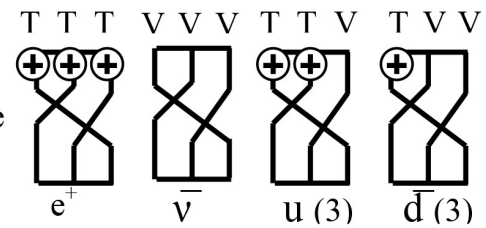

anti-rishon space
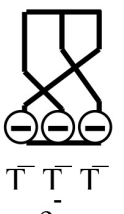

$\mathrm{e}^{-}$

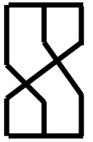

$\mathrm{u}(3)$
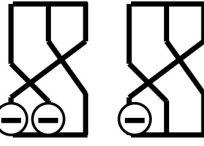

u (3)

$\checkmark$ d (3) right handed space
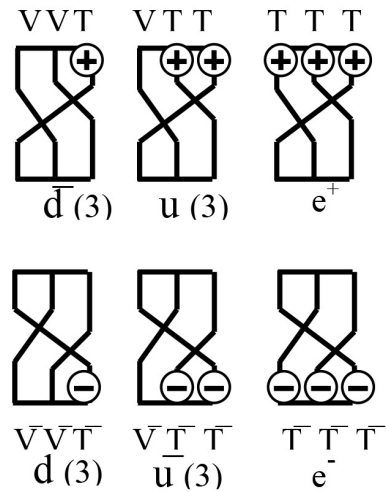

$\mathrm{e}^{-}$

Figure 5. The fermions are represented by the rishon space and helon. Charged fermions have two handedness states each, while $v$ and $\bar{v}$ have only one each. (3) denotes that there are three possible permutations, identified as the quark colors.

$$
\begin{aligned}
& \text { zero-energy groud state space } \stackrel{\text { spontaneous symmetry breaking }}{\longrightarrow} \\
& \text { nonzero-energyscalar Higgs field } \stackrel{\text { massless particle }}{\longrightarrow} \\
& \stackrel{\text { the transitional nonzero-energy Higgs field - particle composite] }}{\stackrel{\text { spontaneous symmetry restoring }}{\longrightarrow} \text { massive particle with the longitudinal }} \\
& \text { component on zero-energy ground state space without the Higgs field }
\end{aligned}
$$

In conventional physics, the nonzero-energy scalar Higgs Field exists permanently in the universe. The problem with such nonzero-energy field is the cosmological constant problem from the huge gravitational effect by the nonzero-energy Higgs field in contrast to the observation [15].

Unlike passive space in conventional physics, the Higgs space [16] as the zero-energy ground state space couples with particles. Attachment space is the space precursor of the Higgs field. Under spontaneous symmetry breaking, attachment space as the active zero-energy ground state space couples with massless particle to form momentarily the transitional non-zero energy Higgs field-particle composite. The Higgs field is momentary and transitional, avoiding the cosmological constant problem. Under spontaneous symmetry restoring, 
the transitional nonzero-energy Higgs field-particle composite is converted into massive particle with the longitudinal component on zero-energy attachment space without the Higgs field as follows.

masslessparticle + zero-energy attachment space $\stackrel{\text { spontaneous symmetry breaking }}{\longrightarrow}$ [the transitional non-zero energy Higgs field - particle composite]

$\stackrel{\text { spontaneous symmetry restoring }}{\longrightarrow}$ massive particle with the longitudinal

component on zero-energy attachment space without the Higgs field

Detachment space is the space precursor of the reverse Higgs field. Unlike the conventional model, detachment space actively couples to massive particle. Under spontaneous symmetry breaking, the coupling of massive particle to zero-energy detachment space produces the transitional nonzero-energy reverse Higgs field-particle composite which under spontaneous symmetry restoring produces massless particle on zero-energy detachment space without the longitudinal component without the reverse Higgs field as follows.

massive particle + zero-energy detachment space $\stackrel{\text { spontaneous symmetry breaking }}{\longrightarrow}$

[the transitional nonzero-energy reverse Higgs field - particle composite]

$\stackrel{\text { spontaneous symmetry restoring }}{\longrightarrow}$ massless particle without the longitudinal

component on zero-energy detachment space without the reverse Higgs field

For the electroweak interaction in the Standard model where the electromagnetic interaction and the weak interaction are combined into one symmetry group, under spontaneous symmetry breaking, the coupling of the massless weak $\mathrm{W}$, weak Z, and electromagnetic A (photon) bosons to zero-energy attachment space produces the transitional nonzero-energy Higgs fields-bosons composites which under partial spontaneous symmetry restoring produce massive $\mathrm{W}$ and $\mathrm{Z}$ bosons on zero-energy attachment space with the longitudinal component without the Higgs field, massless A (photon), and massive Higgs boson as follows.

massless $\mathrm{WZ}+$ zero-energy $\mathrm{WZ}$ attachment space + massless $\mathrm{A}$

+ zero-energy A attachment space $\mathrm{A} \stackrel{\text { spontaneous symmetry breaking }}{\longrightarrow}$

[the transitional nonzero-energy WZ Higgs field - WZ composite]

$+[$ nonzero-energy A Higgs field - A composite $] \stackrel{\text { partial spontaneous symmetry restoring }}{\longrightarrow}$

massive $\mathrm{WZ}$ with the longitudinal component on attachment space without

the Higgs field + massless A + the nonzero energy massive Higgs boson

In terms of mathematical expression, the conventional permanent Higgs field model and the transitional Higgs field model are identical. The interpretations of the mathematical expression are different for the permanent Higgs field model and the transitional Higgs field model. The transitional Higgs field model avoids the cosmological problem in the permanent Higgs field model.

In the Higgs mechanism, gauge bosons are assumed to be massless originally. Elementary fermions (leptons and quarks) can be assumed to be massive originally. However, the observed neutrinos are nearly massless and left-handed only. 
The paper posits that the left handed became massless through the reverse Higgs mechanism. For the symmetrical massive left handed neutrinos and right-handed neutrinos under spontaneous symmetry breaking, the coupling of the massive left handed neutrinos and the massive right handed neutrinos to zero-energy detachment space produces the transitional nonzero-energy reverse Higgs fields-neutrinos composites which under partial spontaneous symmetry restoring produce massless left handed neutrinos on zero-energy detachment space without the longitudinal component without the reverse Higgs field, massive right-handed neutrinos (dark matter), and the massive reverse Higgs boson as follows.

$$
\begin{aligned}
& \text { massive } v_{\mathrm{L}}+\text { zero energy } v_{\mathrm{L}} \text { detachment space }+ \text { massive } v_{\mathrm{R}} \\
& + \text { zero-energy } v_{\mathrm{R}} \text { detachment space } \stackrel{\text { spontaneous symmetry breaking }}{\longrightarrow} \\
& \text { [the transitional nonzero-energy } \left.v_{\mathrm{L}} \text { reverse Higgs field }-v_{\mathrm{L}} \text { composite }\right] \\
& +\left[\text { nonzero-energy } v_{\mathrm{R}} \text { reverse Higgs field }-v_{\mathrm{R}} \text { composite }\right] \\
& \stackrel{\text { partial spontaneous symmetry restoring }}{\longrightarrow} \text { massless } v_{\mathrm{L}} \text { without the longitudinal } \\
& \text { component on det achment space without the Higgs field } \\
& + \text { massive } v_{\mathrm{R}}+\text { the nonzero energy massive reverse Higgs boson }
\end{aligned}
$$

As described in the previous paper [21], the reverse Higgs boson was observed as the two unusual steeply upward-going ultra-high-energy (UHE) cosmic ray events with energies of $\approx 0.6 \mathrm{EeV}[23]$ and $\approx 0.56 \mathrm{EeV}$ [24] in the Antarctic Impulsive Transient Antenna (ANITA) experiment [25]. These shower events have the characteristics of the decay of a tau lepton, which emerges from the surface of the ice, and the tau lepton is explained as the product of a UHE parent tau neutrino by the charged-current interactions with the Earth matter. However, such UHE tau neutrino cannot survive the passage through the Earth. The previous paper posits that the upward-going ANITA events are derived from the cosmic ray of the baryonic-dark matter reverse Higgs boson that travels through the Earth. The calculated value for the reverse Higgs boson is $0.47 \mathrm{EeV}$ in good agreement with the observed 0.56 and 0.6 EeV. As shown in Section 4, dark matter (sterile neutrinos) is part of the periodic table of elementary particles for baryonic matter and dark matter.

\subsection{The Space Structures}

The symmetrical combination of $n$ units of attachment space as 1 and $n$ units of detachment space as 0 brings about three different space structures: binary partition space, miscible space, or binary lattice space as below.

$(1)_{n}$

$+$

$(0)_{n}$<smiles>C=C1CC1</smiles>

$(1)_{n}(0)_{n}$

$(1+0)_{n} \quad$, or

attachment space detachment space binary partition space, miscible space, binary lattice space

Binary partition space, $(1)_{n}(0)_{n}$, consists of two separated continuous phases of multiple quantized units of attachment space and detachment space. In miscible space, $(1+0)_{n}$, attachment space is miscible to detachment space, and there is no separation of attachment space and detachment space. Binary lattice space, (1 
$0)_{n}$, consists of repetitive units of alternative attachment space and detachment space. In conventional physics, space does not couple with particles. In the rest-movement system, space couples with particles.

In binary partition space $(1)_{n}(0)_{n}$, an entity is both in constant motion as standing wave for detachment space and in stationary state as a particle for attachment space, resulting in the wave-particle duality. Such duality can be described by the uncertainty principle. The uncertainty principle for quantum mechanics is expressed as follows.

$$
\sigma_{x} \sigma_{p} \geq \frac{\hbar}{2}
$$

The position, $x$, and momentum, $p$, of a particle cannot be simultaneously measured with arbitrarily high precision. The uncertainty principle requires every physical system to have a zero-point energy (non-zero minimum momentum) and to have a non-zero minimum wavelength as the Planck length. In terms of the binary partition space, detachment space relating to kinetic energy as momentum is $\sigma_{p}$, and attachment space relating to space (wavelength) for a particle is $\sigma_{x}$ In binary partition space, neither detachment space nor attachment space is zero in the uncertainty principle, and detachment space is inversely proportional to attachment space. Quantum mechanics for a particle follows the uncertainty principle defined by binary partition space. Binary partition space $(1)_{n}(0)_{n}$ can also be described by the Schrodinger in quantum mechanics where total energy equals to kinetic energy related to $(0)_{n}$ plus potential energy related to $(1)_{n}$.

In binary partition space, for every detachment space, there is its corresponding adjacent attachment space. Thus, no part of the mass-energy can be irreversibly separated from binary partition space, and no part of a different mass-energy can be incorporated in binary partition space. Binary partition space represents coherence as wavefunction. Binary partition space is for coherent system. Any destruction of the coherence by the addition of a different mass-energy to the mass-energy causes the collapse of binary partition space into miscible space. The collapse is a phase transition from binary partition space to miscible space.

$$
\begin{gathered}
(0)_{n}(1)_{n} \stackrel{\text { collapse }}{\longrightarrow} \\
\text { binary partition space } \\
\text { miscible space }
\end{gathered}
$$

Another way to convert binary partition space into miscible space is gravity. Penrose [26] pointed out that the gravity of a small object is not strong enough to pull different states into one location. On the other hand, the gravity of large object pulls different quantum states into one location to become miscible space. Therefore, a small object without outside interference is always in binary partition space, while a large object is never in binary partition space.

The information in miscible space is contributed by the miscible combination of both attachment space and detachment space, so information can no longer be non-localized. Any value in miscible space is definite and deterministic. All 
observations in terms of measurements bring about the collapse of wavefunction, resulting in miscible space that leads to eigenvalue as definite quantized value. Such collapse corresponds to the appearance of eigenvalue, $E$, by a measurement operator, $H$, on a wavefunction, $\Psi$.

$$
H \Psi=E \Psi
$$

In miscible space, attachment space is miscible to detachment space, and there is no separation of attachment space and detachment space. In miscible space, attachment space contributes zero speed, while detachment space contributes the speed of light. For a moving massive particle consisting of a rest massive part and a massless part, the massive part with rest mass, $m_{0}$, is in attachment space, and the massless part with kinetic energy, $K$, is adjacent to detachment space. The combination of the massive part in attachment space and massless part in detachment leads to the propagation speed in between zero and the speed of light. To maintain the speed of light constant for a moving particle, the time $(t)$ in moving particle has to be dilated, and the length $(L)$ has to be contracted relative to the rest frame.

$$
\begin{aligned}
& t=t_{0} / \sqrt{1-v^{2} / c^{2}}=t_{0} \gamma, \\
& L=L_{0} / \gamma, \\
& E=K+m_{0} c^{2}=\gamma m_{0} c^{2}
\end{aligned}
$$

where $\gamma=1 /\left(1-v^{2} / c^{2}\right)^{1 / 2}$ is the Lorentz factor for time dilation, and length contraction, $E$ is the total energy, and $K$ is the kinetic energy. Binary lattice space, (1 0)n, as the space of virtual gauge rishon boson in quantum field theory will be described in Section 4.

\section{Cosmology}

The paper posits that the cyclic universe cosmology in the multiverse involves the split of the membrane 11D spacetime into the $1 \mathrm{D}$ eleventh dimension orbifold interval space to form gravity, the $6 \mathrm{D}$ discrete interior rishon space (TTT-VVV for positron-neutrino or TTV-TVV for u-d quarks) to form the Standard Model, the 3D Higgs space (attachment space to attach matter or detachment space to detach matter) to form the Higgs or reverse Higgs field, and 1D Einstein time to be shared by all spaces.

\subsection{The Multiverse}

This paper posits that the origin of the split is derived from the cyclic universe cosmology in the multiverse. The multiverse consists of the zero-energy inter-universal void with $4 \mathrm{D}$ detachment space and the multiple $11 \mathrm{D}$ positive-energy membrane and negative-energy anti-membrane dual universes with 11D attachment space which has zero total energy. Detachment space in the inter-universal void prevents the collision of the 11D dual universes, while attachment space in the dual universe allows the existence of mass-energy in the space of the dual universe. The dual universe with zero total energy can emerge 
from the zero-energy inter-universe. Each of the positive-energy 11D universe and the negative-energy $11 \mathrm{D}$ universe does not conserve the energy-mass conservation, but the energy sum of the positive-energy $11 \mathrm{D}$ universe and the negative-energy $11 \mathrm{D}$ universe is zero which conserves the energy-mass conservation.

The cyclic universe cosmology starts with the zero-energy inter-universal void and the positive-negative energy $11 \mathrm{D}$ membrane dual universe which is split into four 10D string branes as the dual two-string branes, including the positive-energy weak-gravity string brane, the negative-energy weak-gravity string brane, the positive-energy strong-gravity string brane, and the negative-energy strong-gravity string brane in the $11 \mathrm{D}$ dual universe bulk. The positive-energy weak string brane contains matter, while the negative-energy string brane contains antimatter. The matter-antimatter balance occurs in the balance between the matter in the positive-energy weak-gravity string brane and the antimatter in the negative-energy weak-gravity string brane as in Figure 6. Each brane has the matter-antimatter imbalance initially. Afterward, all subsequent events in each brane have the matter-antimatter balance for CP symmetry. The strong string branes are dominated by gravity. All four branes have equal mass-energy. The $1 \mathrm{D}$ interval space is between the $10 \mathrm{D}$ strong-gravity string brane and the $10 \mathrm{D}$ weak-gravity string brane in the 11D membrane bulk.

In conventional physics, space-time dimension numbers are fixed. Compactization is required to account for the observed 4D [2]. As described previously [19] [20], the space-time dimension numbers oscillate reversibly between 10D and $4 \mathrm{D}$ reversibly dimension by dimension without compactization. The oscillating space-time numbers from $10 \mathrm{D}$ to $4 \mathrm{D}$ relate to varying speed of light. Varying speed of light has been proposed to explain the horizon problem of cosmology [27] [28]. J. D. Barrow [29] proposes that the time dependent speed of light varies as some power of the expansion scale factor $a$ in such way that

$$
c(t)=c_{0} a^{n},
$$

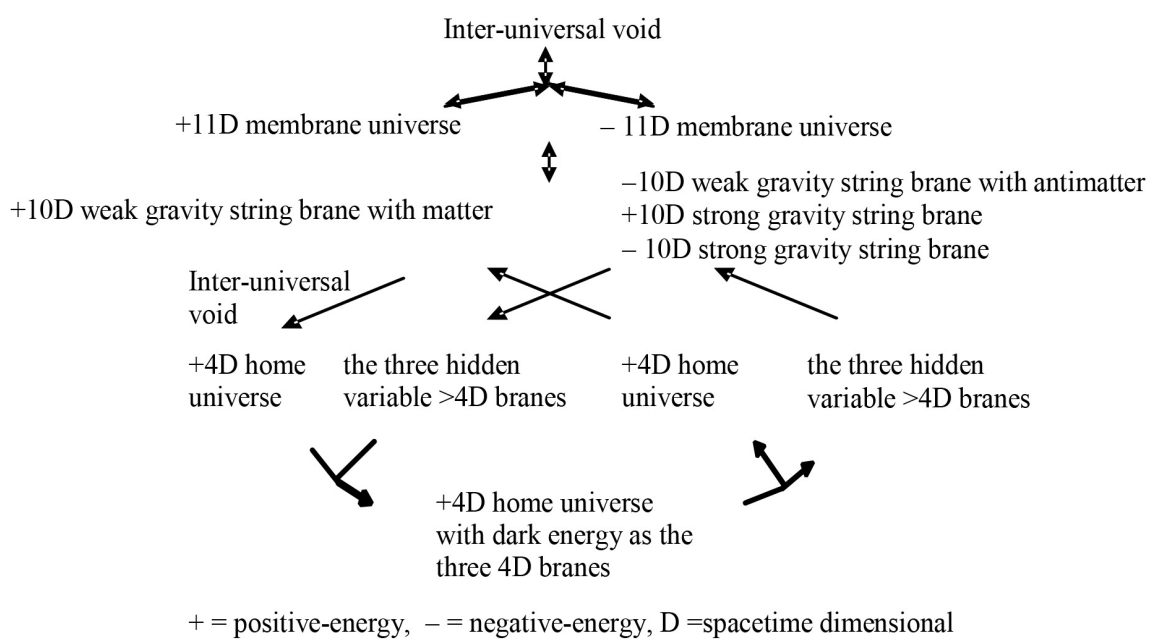

Figure 6. The reversible cyclic universe. 
where $c$ is the speed of light and $n$ are constants. The increase of speed of light is continuous.

In this paper, the speed of light is invariant in a constant space-time dimension number, and the speed of light varies with varying space-time dimension number from 4 to 11 as follows.

$$
c_{\mathrm{D}}=c / \alpha^{\mathrm{D}-4},
$$

where $c$ is the observed speed of light in the $4 \mathrm{D}$ space-time, $c_{\mathrm{D}}$ is the quantized varying speed of light in space-time dimension number, $D$, from 4 to 10 , and $\alpha$ is the fine structure constant for electromagnetism. The speed of light increases with the increasing space-time dimension number D. Since the speed of light for $>4 \mathrm{D}$ particle is greater than the speed of light for $4 \mathrm{D}$ particle, the observation of $>4 \mathrm{D}$ particles by $4 \mathrm{D}$ particles violates casualty. Thus, $>4 \mathrm{D}$ particles are hidden particles with respect to $4 \mathrm{D}$ particles. Particles with different space-time dimensions are transparent and oblivious to one another, and separate from one another if possible.

As described previously [19] [20], the particle oscillation between 10D and 10D through $4 \mathrm{D}$ involves mass dimension (denoted as $\mathrm{d}$ ) to represent the mass. In the initial condition for the oscillation, $\mathrm{D}+\mathrm{d}=14$ where $\mathrm{D}$ and $\mathrm{d}$ are between 4 and 10. For an example, a dimension has a dual spacetime-mass dimension numbers of $10 \mathrm{D} 4 \mathrm{~d}$ or $4 \mathrm{D} 10 \mathrm{~d}$. The transformations for oscillating dimension number between 10D and 4D consist of the varying speed of light dimensional (VSLD) transformation for spacetime dimension $\mathrm{D}$ and the varying supersymmetry dimensional (VSD) transformation for mass dimension d. For the VSLD transformation for D, $E=M_{0} c^{2}$ modified by Equation (13) is expressed as

$$
\begin{gathered}
E=M_{0} c_{\mathrm{D}}^{2}=M_{0} \cdot\left(c^{2} / \alpha^{2(\mathrm{D}-4)}\right), \\
M_{0, \mathrm{D}, \mathrm{d}}=M_{0, \mathrm{D}-n, \mathrm{~d}+n} \alpha^{2 n}, \\
E_{\text {vacuum, } \mathrm{D}}=E-M_{0, \mathrm{D}} c^{2}, \\
\mathrm{D}, \mathrm{d} \stackrel{\text { vsLD transformation }}{\longrightarrow}(\mathrm{D} \mp n),(\mathrm{d} \pm n)
\end{gathered}
$$

where $c_{\mathrm{D}}$ is the quantized varying speed of light in space-time dimension number, D, from 4 to $10, c$ is the observed speed of light in the $4 \mathrm{D}$ space-time, $\alpha$ is the fine structure constant for electromagnetism, $E$ is energy, $M_{0}$ is rest mass, $\mathrm{D}$ is the space-time dimension number from 4 to $10, \mathrm{~d}$ is the mass dimension number from 4 to $10, n$ is an integer, and $E_{\text {vacuum }}=$ vacuum energy. From Equation (14), 10D has the lowest rest mass, and $4 \mathrm{D}$ has the highest rest mass. According to the calculation from Equation (15), the rest mass of $4 \mathrm{D}$ is $1 / \alpha^{12} \approx$ $137^{12}$ times of the mass of 10D. From Equation (16), 10D has the highest vacuum energy, while $4 \mathrm{D}$ particle has zero vacuum energy. A particle with $10 \mathrm{D}$ is transformed to a particle with $4 \mathrm{D}$ from Equation (17) through the VSLD transformation. Spacetime dimension number decreases with decreasing speed of light, decreasing vacuum energy, and increasing rest mass. The $4 \mathrm{D}$ and the $10 \mathrm{D}$ have zero and the highest vacuum energies, respectively. 
In the normal supersymmetry transformation, the repeated application of the fermion-boson supersymmetry transformation carries over a boson (or fermion) from one point to the same boson (or fermion) at another point at the same mass, resulting in translation without changing mass. Under the varying supersymmetry dimensional (VSD) transformation, the repeated application of the fermion-boson supersymmetry transformation carries over a boson from one point to the boson at another point at different mass dimension number at different mass, resulting in translation and fractionalization or condensation. The repeated VSD transformation carries over a boson $\mathrm{B}_{\mathrm{d}}$ into a fermion $F_{\mathrm{d}}$ and a fermion $F_{\mathrm{d}}$ to a boson $B_{\mathrm{d}-1}$, which can be expressed as follows.

$$
\begin{gathered}
M_{\mathrm{d}, \mathrm{F}}=M_{\mathrm{d}, \mathrm{B}} \alpha_{\mathrm{d}, \mathrm{B}}, \\
M_{\mathrm{d}-1, \mathrm{~B}}=M_{\mathrm{d}, \mathrm{F}} \alpha_{\mathrm{d}, \mathrm{F}},
\end{gathered}
$$

where $M_{\mathrm{d}, \mathrm{B}}$ and $M_{\mathrm{d}, \mathrm{F}}$ are the masses for a boson and a fermion, respectively, $\mathrm{d}$ is the mass dimension number, and $\alpha_{\mathrm{d}, \mathrm{B}}$ or $\alpha_{\mathrm{d}, \mathrm{F}}$ is the fine structure constant that is the ratio between the masses of a boson and its fermionic partner. where $M_{\mathrm{d}, \mathrm{B}}$ and $M_{\mathrm{d}, \mathrm{F}}$ are the masses for a boson and a fermion, respectively, $\mathrm{d}$ is the mass dimension number, and $\alpha_{\mathrm{d}, \mathrm{B}}$ or $\alpha_{\mathrm{d}, \mathrm{F}}$ is the fine structure constant that is the ratio between the masses of a boson and its fermionic partner. Assuming $\alpha$ 's are the same, it can be expressed as

$$
M_{\mathrm{d}, \mathrm{B}}=M_{\mathrm{d}+1, \mathrm{~B}} \alpha_{\mathrm{d}+1}^{2} \text {. }
$$

The varying supersymmetry transformation involves the translation and fractionalization from $d$ to $d-1$ or condensation from $d$ to $d+1$ at the same $D$. The translation and fractionalization-condensation account for the cosmic expansion-contraction for the oscillating universes.

The transformation during the oscillation between $10 \mathrm{D}$ particle and $4 \mathrm{D}$ particle involves the stepwise two-step transformation consisting of the VSLD transformation and the VSD transformation. The VSLD transformation involves the transformation of spacetime dimension, D whose mass increases with decreasing $\mathrm{D}$ for the decrease in vacuum energy. The VSD transformation involves the transformation of the mass dimension number, $d$ whose mass decreases with decreasing $\mathrm{d}$ for the fractionalization of particle. The oscillating dimension number transformation between 10D4d and 10D4d through 4D4d involves both the VSLD transformation and the VSD transformation as the stepwise two-step transformation as follows.

stepwise two-step varying transformation

$$
\begin{aligned}
& \text { (1) } \mathrm{D}, \mathrm{d} \stackrel{\mathrm{VSLD} \text { transformation }}{\stackrel{\mathrm{VSD} \text { transformation }}{\longrightarrow}}(\mathrm{D} \mp 1),(\mathrm{d} \pm 1) \\
& \text { (2) } \mathrm{D}, \mathrm{d} \pm 1)
\end{aligned}
$$

The repetitive stepwise two-step dimension number oscillation between 10D4d and 10D4d through 4D4d as follows.

$$
\begin{aligned}
& 10 \mathrm{D} 4 \mathrm{~d} \rightarrow \text { 9D5d } \rightarrow \text { 9D4d } \rightarrow \text { 8D5d } \rightarrow \text { 8D4d } \rightarrow \text { 7D5d } \rightarrow \text { 7D4d } \rightarrow \text { 6D5d } \\
& \rightarrow \text { 6D4d } \rightarrow \text { 5D5d } \rightarrow \text { 5D4d } \rightarrow \text { 4D5d } \rightarrow \text { 4D4d } \rightarrow \text { 5D4d } \rightarrow \text { 5D5d } \rightarrow \text { 6D4d } \\
& \rightarrow \text { 6D5d } \rightarrow \text { 7D4d } \rightarrow \text { 7D5d } \rightarrow \text { 8D4d } \rightarrow \text { 8D5d } \rightarrow \text { 9D4d } \rightarrow \text { 9D5d } \rightarrow \text { 10D4d }
\end{aligned}
$$


As described previously [19] [20], the oscillation between 10D and 4D results in the reversible cyclic fractionalization-contraction for the reversible cyclic expansion-contraction of the universe.

\subsection{The Home Universe}

To form the home universe where we inhabit, the 10D positive-energy weak-gravity string brane with attachment space absorbed the zero-energy 4D inter-universal void with $4 \mathrm{D}$ detachment space, resulting in the home universe which contains the Higgs space with both attachment space for rest mass and detachment space for kinetic energy. The home universe resulted from the transformation from a $10 \mathrm{D}$ spacetime brane into a $4 \mathrm{D}$ spacetime universe by transforming $6 \mathrm{D}$ connected exterior space into $6 \mathrm{D}$ discrete interior rishon space. The other three $10 \mathrm{D}$ string branes did not absorb detachment space. From $10^{-36}$ seconds after the entrance of detachment space to the time between $10^{-33}$ and $10^{-32}$ seconds after the entrance of detachment space, four important events occurred to transform the $10 \mathrm{D}$ positive-energy weak-gravity string brane into the $4 \mathrm{D}$ home universe where we inhabit. The four events are cosmic inflation derived from the transformation from $10 \mathrm{D} 4 \mathrm{~d}$ to $4 \mathrm{D} 10 \mathrm{~d}$, the rishon space formation by transforming $6 \mathrm{D}$ connected exterior space into $6 \mathrm{D}$ discrete interior rishon space, the Higgs space formation by the combination of attachment space and detachment space, and the Extend Standard Model from the periodic table of elementary particles based on the seven mass dimensions.

\subsubsection{Cosmic Inflation}

Cosmic inflation is derived the transformation from 10D4d to $4 \mathrm{D} 10 \mathrm{~d}$ immediately. Calculated from Equation (15), the rest mass of $4 \mathrm{D} 10 \mathrm{~d}$ is $M_{0,10}=M_{0,4} / \alpha^{2(10-4)} \approx 137^{12}$ times of the rest mass of $10 \mathrm{D} 4 \mathrm{~d}$, resulting in cosmic inflation as the exponential expansion of space from the high vacuum energy $10 \mathrm{D} 4 \mathrm{~d}$ to the zero vacuum energy $4 \mathrm{D} 10 \mathrm{~d}$ as follows.

inflation

10D4d spacetime + 1D interval space for gravity $\stackrel{\text { quick VSLD transformation }}{\longrightarrow}$

4D10d spacetime +1D interval space for gravity

\subsubsection{The Rishon Space Formation}

The home universe resulted from the transformation from a $10 \mathrm{D}$ spacetime brane into a $4 \mathrm{D}$ spacetime universe by transforming $6 \mathrm{D}$ connected exterior space into $6 \mathrm{D}$ discrete interior rishon space as follows.

$10 \mathrm{D}$ connected exterior spacetime for string $\stackrel{\text { from } 10 \mathrm{D} \text { to } 4 \mathrm{D}}{\longrightarrow}$

$4 \mathrm{D}$ connected exterior spacetime $+6 \mathrm{D}$ discrete interior rishon space(TTTVVV) for e- $v$ or 4D connected exterior spacetime $+6 \mathrm{D}$ discrete interior rishon space(TTVTVV) for $\mathrm{u}-\overline{\mathrm{d}} \longrightarrow$

TTT for e and VVV for $v$ or TTV for $u$ and TVV for $\overline{\mathrm{d}}$ in $4 \mathrm{D}$ connected exterior spacetime 
The rishon space formation produces the discrete space in addition to connected space. All elementary particles are in discrete space in addition to connected space, while gravity in the interval space continues to be in connected exterior space without discrete interior space for flavor, charge, isospin, and color.

\subsubsection{The Higgs Space Formation}

As discussed in Section 3, the space structures as the mixed Higgs spaces as the combinations of $n$ units of attachment space (denoted as 1 from the positive-energy weak-gravity string brane) and $n$ units of detachment space (denoted as 0 from the inter-universal void) consist of binary partition space, $(1)_{n}(0)_{n}$, as the space of wave-particle duality, binary miscible space, $(1+0)_{n}$, as the space of relativity, and binary lattice space, $(10)_{m}$, as the space of virtual gauge boson in quantum field theory. Section 3 describes binary partition space and binary miscible space, and binary lattice space is described as follows.

Binary lattice space is derived from the slicing of mass dimension. Bounias and Krasnoholovets [30] propose that the reduction of dimension can be done by slicing dimension, such as slicing 3 space dimension object (block) into infinite units of 2 space dimension objects (sheets). Cosmic inflation involves the transformation from 10D4d with high vacuum energy into $4 \mathrm{D} 10 \mathrm{~d}$ with zero vacuum energy at once. 4D10d particle was sliced into six different particles: 4D9d, 4D8d, 4D7d, 4D6d,4D5d, and 4D4d equally by mass. Baryonic matter is $4 \mathrm{D} 4 \mathrm{~d}$ which is the lowest spacetime-mass dimension from Equation (22), while dark matter consisted of the other five types of particles (4D9d, 4D8d, 4D7d, 4D6d, and 4D5d) as follows.

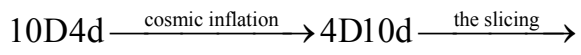

baryonic matter (4D4d) + dark matter (4D5d, 4D6d, 4D7d, 4D8d, 4D9d)

As a result, the mass ratio of dark matter to baryonic matter is 5 to 1 in excellent agreement with the observed 4.98 to 1 derived from the observed $4.56 \%$ and 22.7\% for baryonic matter and dark matter, respectively [19] [31].

The $10 \mathrm{~d}$ (mass dimension) particle in attachment space denoted as 1 was sliced by detachment space denoted as 0 . For example, the slicing of $10 \mathrm{~d}$ particle into $4 \mathrm{~d}$ particle is as follows.

$$
\begin{gathered}
1_{10} \quad \stackrel{\text { slicing }}{\longrightarrow} 1_{4} \quad \sum_{\mathrm{d}=5}^{10}\left(\begin{array}{ll}
0_{4} & 1_{4}
\end{array}\right)_{n, \mathrm{~d}} \\
\text { 10d particle } \quad \text { 4d core particle } \\
\text { binary lattice space }
\end{gathered}
$$

where $1_{10}$ is $10 \mathrm{~d}$ particle, $1_{4}$ is $4 \mathrm{~d}$ particle, $\mathrm{d}$ is the mass dimension number of the dimension to be sliced, $\mathrm{n}$ as the number of slices for each dimension, and $\left(0_{4} 1_{4}\right)_{n}$ is binary lattice space with repetitive units of alternative $4 \mathrm{~d}$ attachment space and $4 \mathrm{~d}$ detachment space. For $4 \mathrm{~d}$ particle starting from $10 \mathrm{~d}$ particle, the mass dimension number of the dimension to be sliced is from $d=5$ to $d=10$. Each mass dimension is sliced into infinite quantized units $(n=\infty)$ of binary lattice space, $\left(0_{4}, 1_{4}\right)_{\infty}$. For $4 \mathrm{~d}$ particle, the $4 \mathrm{~d}$ core particle is surrounded by 6 types (from $d=5$ to $d=10$ ) of infinite quantized units of binary lattice space. Such in- 
finite quantized units of binary lattice space represent the infinite units $(n=\infty)$ of separate energy virtual orbitals (probabilities) for virtual particles in a gauge force field. There are six gauge fields from $d=5$ to $d=10$. The three force fields, electromagnetism, the strong force, and the weak force are represented by $\mathrm{d}=5$, 6 , and 7 , respectively. For dark matter, $\mathrm{d}=5$ for electromagnetism is not sliced, so dark matter does not have electromagnetism at $d=5$, resulting in the darkness due to the absence of photon from electromagnetism.

The Higgs space produces the Higgs mechanism and the space structures. Quantum mechanics is derived from the Higgs space, so the simultaneous occurrence of cosmic inflation and quantum mechanism produced cosmic quantum fluctuation in the home universe.

\subsubsection{The Extend Standard Model}

The Extend Standard Model includes leptons, quarks, gauge bosons, gravity, dark matter, and dark energy.

\section{The Seven Mass Dimensions}

The masses in the Extend Standard Model are based on the seven mass dimensions. Cosmic inflation produced 4D10d spacetime and 1D interval space for gravity which were transformed into $4 \mathrm{D} 4 \mathrm{~d}$ spacetime as the lowest spacetime-mass dimension from Equation (22) $+1 \mathrm{D}$ interval space for gravity +6 mass dimensions for non-gravity +1 mass dimension for gravity. The combination results in 4D4d spacetime $+1 \mathrm{D}$ interval space for gravity +7 mass dimensions as Equation (27) and in Figure 7.

10D4d +1D interval space for gravity $\stackrel{\text { cosmic inflation }}{\longrightarrow}$

$4 \mathrm{D} 10 \mathrm{~d}$ spacetime $+1 \mathrm{D}$ interval space for gravity $\longrightarrow 4 \mathrm{D} 4 \mathrm{~d}$ spacetime

$+1 \mathrm{D}$ interval space for gravity +6 mass dimensions for non-gravity

+1 mass dimension for gravity $\stackrel{\text { combinasion }}{\longrightarrow} 4 \mathrm{D} 4 \mathrm{~d}$ spacetime

$+1 \mathrm{D}$ interval space for gravity +7 mass dimensions

The seven mass dimensions are arranged as $\mathrm{F}_{5} \mathrm{~B}_{5} \mathrm{~F}_{6} \mathrm{~B}_{6} \mathrm{~F}_{7} \mathrm{~B}_{7} \mathrm{~F}_{8} \mathrm{~B}_{8} \mathrm{~F}_{9} \mathrm{~B}_{9} \mathrm{~F}_{10} \mathrm{~B}_{10}$ $F_{11} B_{11}$, where $F_{d}$ and $B_{d}$ are mass dimensional fermion and mass dimensional boson, respectively. Under the varying supersymmetry dimensional (VSD) transformation, the mass of mass dimensional fermion and the mass of mass dimensional boson are related to each other with three simple formulas as follows.

$$
\begin{aligned}
& M_{\mathrm{d}, \mathrm{B}}=M_{\mathrm{d}, \mathrm{F}} / \alpha_{\mathrm{d}} \\
& M_{\mathrm{d}+1, \mathrm{~F}}=M_{\mathrm{d}, B} / \alpha_{\mathrm{d}+1} \\
& M_{\mathrm{d}+1, \mathrm{~B}}=M_{\mathrm{d}, \mathrm{B}} / \alpha_{\mathrm{d}+1}^{2},
\end{aligned}
$$

where $d$ is the mass dimension number, $F$ is fermion, and $B$ is boson. Each dimension has its own $\alpha_{\mathrm{d}}$, and all $\alpha_{\mathrm{d}}$ 's except $\alpha_{7}\left(\alpha_{w}\right)$ of the seventh dimension (weak interaction) are equal to $\alpha$, the fine structure constant of electromagnetism.

For baryonic matter, with electromagnetism at $\mathrm{d}=5$, electron appears in addition to neutrino. Without electromagnetism at $d=5$, dark matter does not 


$$
\mathrm{d}=\begin{array}{l|l|l|l|l|l|l|}
5 & 6 & 7 & 8 & 9 & 10 & 11
\end{array}
$$

Figure 7. The 7 mass dimensions.

have charge particle. For baryonic matter, to represent the mixed rishon space (TTV-TVV) for charge u-d quarks, the additional seven mass dimensions as the "auxiliary mass dimensions" (a's) to represent quarks and unstable leptons in addition to the seven "principal mass dimensions" (d's) to represent neutrino-electron and gauge bosons as in Figure 8.

\section{The Periodic Table of Elementary Particles}

The periodic table of elementary particles [21] [22] is based on the seven principal mass dimensions (d's) for stable baryonic matter leptons (electron and neutrinos), gauge bosons (all forces), gravity, and dark matter (five sterile dark matter neutrinos) and the seven auxiliary mass dimensions (a's) for unstable leptons (muon and tau) and quarks (d, u, s, c, b, and t) as in Figure 8 and Table 3.

In the periodic table of elementary particles, the five dark matter particles are derived from Equation (24). Without electromagnetism at $\mathrm{d}=5$, dark matter does not have charge particle, and has to be neutrinos. Initially derived from Equation (25) and the symmetry between dark matter and baryonic matter, there were five dark matter massive right-handed neutrinos and one baryonic matter massive left-handed neutrino. Through the reverse Higgs mechanism as Equation (6), the left-handed neutrino becomes massless, while the right-handed neutrinos as sterile dark matter neutrinos remain massive. The reverse Higgs boson was observed [21]. All neutrinos and electron as well as gauge bosons are in the principal mass dimensions. All quarks and unstable leptons are in the auxiliary mass dimensions. The three generations of baryonic matter lepton-quark is the maximum generations allowed for the seven principal dimensions and the seven auxiliary dimensions.

\section{Gauge Bosons}

In the periodic table of elementary particles, the given observed masses are the mass of electron for $\mathrm{F}_{6}$ and the mass of $\mathrm{Z}$ boson for $\mathrm{B}_{7}$. From Equations (28) and (30), $\alpha_{w}=\alpha_{7}=\alpha$ of week interaction $=\left(M_{\mathrm{B}_{6}} / M_{\mathrm{B}_{7}}\right)^{1 / 2}=\left(M_{\mathrm{F}_{6}} / \alpha / M_{\mathrm{B}_{7}}\right)^{1 / 2}=$ $\left(M_{e} / \alpha / M_{Z}\right)^{1 / 2}=0.02771$. Therefore, the masses of gauge bosons are as in Table 4.

The lowest energy gauge boson $\left(B_{5}\right)$ at $d=5$ is the Coulomb field for electromagnetism. The second gauge lowest boson $\left(B_{6}\right)$ at $d=6$ is basic gluon $\left(g^{*}=70\right.$ $\mathrm{MeV} \approx$ one half of pion) is the strong force as the nuclear force in the pion theory [32] where pions mediate the strong interaction at long enough distances (longer than the nucleon radius) or low enough energies. $B_{6}$ is denoted as basic gluon, $\mathrm{g}^{\star}$. At short enough distances (shorter than the nucleon radius) or high 
Stable Baryonic Matter Leptons

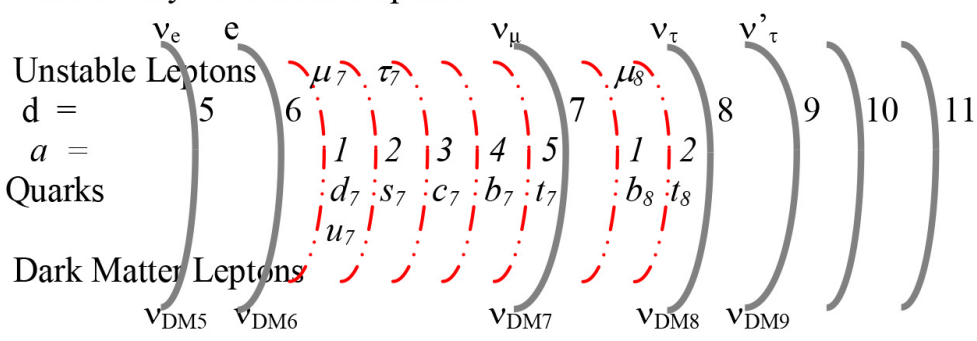

Figure 8. The seven principal mass dimensions (solid lines) denoted by the principal mass dimension number $\mathrm{d}$ and the seven auxiliary mass dimensions (dash-dotted lines) denoted by the auxiliary mass dimension number $a$.

Table 3. The periodic table of elementary particles for baryonic matter and dark matter $\mathrm{d}=$ principal mass dimension number, $\mathrm{a}=$ auxiliary mass dimension number, $\mathrm{DM}=$ dark matter, $\mathrm{BM}=$ baryonic matter.

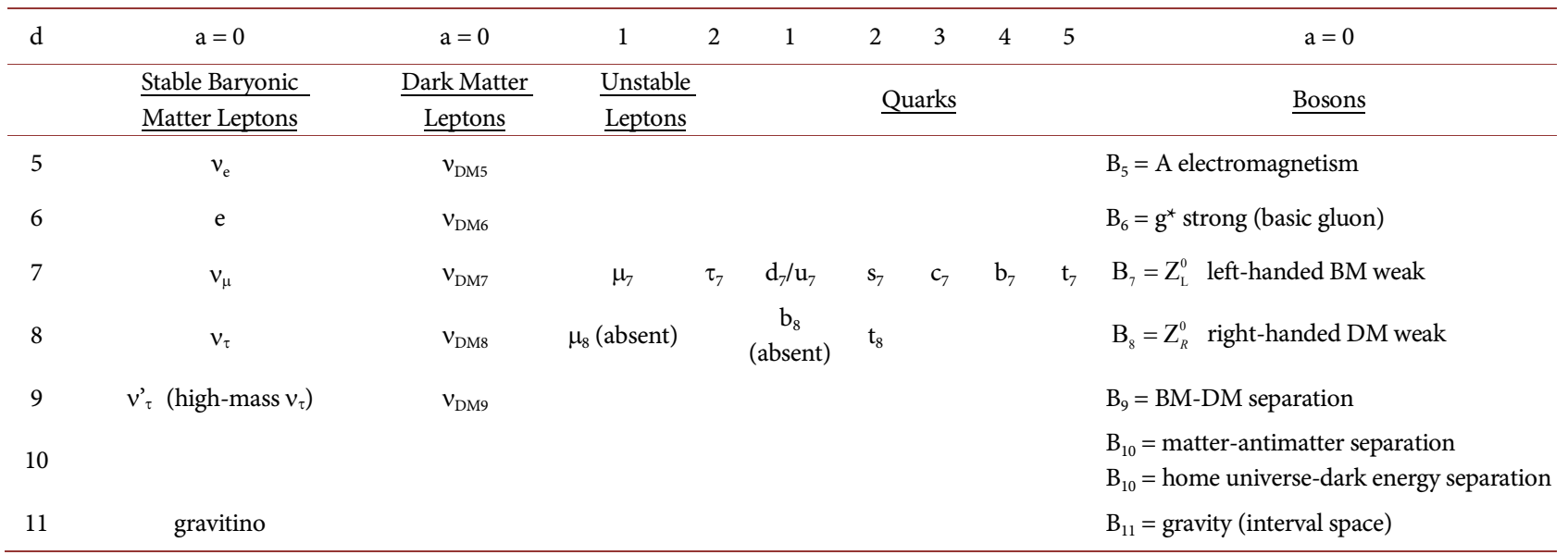

Table 4. The masses of the principal mass dimensions (gauge bosons).

\begin{tabular}{ccccc}
\hline $\mathrm{B}_{\mathrm{d}}$ & $\mathrm{M}_{\mathrm{d}}$ & $\mathrm{GeV}$ (calculated) & Gauge boson & Interaction \\
\hline $\mathrm{B}_{5}$ & $M_{e} a$ & $3.7 \times 10^{-6}$ & $\mathrm{~A}=$ photon & Electromagnetic \\
$\mathrm{B}_{6}$ & $M_{e} / a$ & $7 \times 10^{-2}(70.02 \mathrm{MeV})$ & $\mathrm{g}^{*}=$ basic gluon & Strong \\
$\mathrm{B}_{7}$ & $M_{Z}=M_{\mathrm{B}_{6}} / \alpha_{w}^{2}$ & 91.1876 (given) & $\mathrm{Z}_{\mathrm{L}}$ & weak (left) for baryonic matter \\
$\mathrm{B}_{8}$ & $M_{7} / a^{2}=M_{Z} / \alpha^{2}$ & $1.71 \times 10^{6}$ & $\mathrm{Z}_{\mathrm{R}}$ & weak (right) for dark matter \\
$\mathrm{B}_{9}$ & $M_{8} / a^{2}=M_{Z} / \alpha^{4}$ & $3.22 \times 10^{10}$ & & baryonic matter-dark matter separation \\
$\mathrm{B}_{10}$ & $M_{9} / a^{2}=M_{Z} / \alpha^{6}$ & $6.04 \times 10^{14}$ & & matter-antimatter separation \\
$\mathrm{B}_{11}$ & $M_{10} / a^{2}=M_{Z} / \alpha^{8}$ & $1.13 \times 10^{19}$ & $\mathrm{G}$ & home universe-dark energy separation \\
\end{tabular}

enough energies, gluons emerge to confine fractional charge quarks. Fractional charge quarks are confined by gluons in QCD (quantum chromodynamics). No isolated fractional charge quark is allowed, and only collective integer charge quark composites are allowed. In general, collective fractional charges are confined by the short-distance confinement force field where the sum of the collec- 
tive fractional charges is integer [33]. As a result, fractional charges are confined and collective. The confinement force field includes gluons for collective fractional charge quarks in hadrons and the magnetic flux quanta for collective fractional charge quasiparticles in the fractional quantum Hall effect (FQHE) [34] [35] [36].

The third lowest boson $\left(B_{7}\right)$ at $d=7$ is $Z_{L}$ for the left-handed weak interaction among leptons and quarks. Spontaneous symmetry breaking produces massive weak bosons, massless photon and the Higgs boson as Equation (5). Massive weak bosons produce short-distance interaction. $B_{8}$ at $d=8$ is $Z_{R}$ for the right-handed weak interaction among dark matter neutrinos as dark matter neutrino oscillation. The symmetry between $\mathrm{Z}_{\mathrm{R}}$ and $\mathrm{Z}_{\mathrm{L}}$ provides the neutrino oscillation for both baryonic matter neutrinos [37] and dark matter neutrinos.

At $\mathrm{B}_{9}$, baryonic matter and dark matter are separated. Above $\mathrm{B}_{9}$, there is no baryonic matter and dark matter before the slicing as in Equation (25). $B_{9}$ as the gauge boson represents the weak short-distance repulsive Yukawa force [38] between baryonic matter and dark matter. Long-distance attractive gravity among all particles in a random mixture of dark matter particles and baryonic matter particles overcomes the weak short-distance repulsive force between dark matter particles and baryonic matter particles. However, a large amount of homogeneous dark matter particles repulses a large amount of homogeneous baryonic matter particles in the short-distance interfacial region between a large amount of homogeneous dark matter particles and a large amount of homogeneous baryonic matter particles. This repulsive force is same as the Yukawa force for baryonic matter [39] [40] in the Moffat's Modified Gravity (MOG) theory, and explains the evolution of galaxy [41]. This repulsive force prevents the catch and the direct detection of dark matter particles on earth with a large amount of homogeneous baryonic matter particles.

$B_{10}$ at $d=10$ is for the matter-antimatter separation. At $B_{10}$, matter is in our home universe as the positive energy $10 \mathrm{D}$ string brane, while antimatter is in the negative energy $10 \mathrm{D}$ string brane. Above $\mathrm{B}_{10}$, there is no string brane as in Figure 6. As in Figure 6, $B_{10}$ at $d=10$ is also for the home universe-dark energy separation. Above $B_{10}$, there is no separation between the home universe and the three string branes. $B_{10}$ as the gauge boson represents the repulsive force between matter in the home universe and antimatter in the negative-energy weak gravity brane and between the home universe and dark energy in the three branes.

$B_{11}$ is for gravity in the interval space. $F_{11}\left(8.275 \times 10^{16} \mathrm{GeV}\right)$ relates to spin $3 / 2$ gravitino, while $\mathrm{B}_{11}\left(1.134 \times 10^{19} \mathrm{GeV}\right)$ relates to spin 2 graviton. In supersymmetry, gravitino and graviton mediate the supersymmetry between fermion and boson in space dimension and gravitation. There are 11 space dimensions in the 11 spacetime dimensional membrane. As a result, the supersymmetry involves $11 \mathrm{~F}_{11}+\mathrm{B}_{11}$, which is equal to $1.225 \times 10^{19} \mathrm{GeV}$ in excellent agreement with the Planck mass $\left(1.221 \times 10^{19} \mathrm{GeV}\right)$ derived from observed gravity as $(\hbar \mathrm{c} / \mathrm{G})^{1 / 2}$ where $\mathrm{c}$ is the speed of light, $\mathrm{G}$ is the gravitational constant, and $\mathrm{h}$ is the reduced Planck 
constant. Electromagnetism and gravity are long-distances forces, and all other forces are short-distance.

\section{Leptons and Quarks}

The lepton mass formula and the quark mass formula are derived from the incorporation of basic gluon $\left(\mathrm{g}^{*}=\mathrm{B}_{6}=70 \mathrm{MeV}\right)$ to electron. The incorporation of basic gluon as flux quanta follows the the composite fermion theory for the FQHE (fractional quantum Hall effect) [42] [43]. In the composite fermion model for FQHE, the formation of composite fermion is through the attachment of an even number of magnetic flux quanta to electron, while the formation of composite boson is through the attachment of an odd number of magnetic flux quanta to electron. In the same way, the formation of composite fermion is through the attachment of an even number of basic gluons to electron, while the formation of composite boson is through the attachment of an odd number of basic gluons to electron. The formation of composite boson is equal to the formation of composite di-leptons, so the formation of composite lepton is through the attachment of one half of an odd number of basic gluons to electron. As a result, the muon $(\mu)$ mass formula is as follows.

$$
M_{\mu_{7}}=M_{e}+3 M_{\mathrm{g}^{*}} / 2=M_{e}+3 M_{e} / 2 \alpha=105.5488 \mathrm{MeV},
$$

which is in excellent agreement with the observed $105.6584 \mathrm{MeV}$ [44] for the mass of muon. The masses of leptons follow the Barut lepton mass formula [45] as follows.

$$
M_{\text {lepton }}=M_{e}+\frac{3 M_{e}}{2 \alpha} \sum_{a=0}^{n} a^{4},
$$

where $a=0,1$, and 2 are for $e, \mu_{7}$, and $\tau_{7}$, respectively. The calculated mass of $\tau_{7}$ is $1786.2 \mathrm{MeV}$ in good agreement with the observed mass as $1776.82 \mathrm{MeV}$. According to Barut, the second term, $\sum^{n} a^{4}$ of the mass formula is for the Bohr-Sommerfeld quantization for a chäge-dipole interaction in a circular orbit. The more precise calculated mass of $\tau$ for the tau lepton mass formula is as follows.

$$
\begin{aligned}
M_{\tau} & =M_{e}+\left(\frac{3 M_{e}}{2 \alpha}-M_{e}\right) \sum 2^{4} \\
& =M_{e}+\left(17 \frac{3 M_{e}}{2 \alpha}-17 M_{e}\right) \\
& =1777.47 \mathrm{MeV}
\end{aligned}
$$

which is in excellent agreement with observed $1776.82 \mathrm{MeV}$, and means that during this dipole-interaction in a circular orbit for $\tau$, an electron with total mass of $17 M_{e}$ is lost. $17 M_{e}$ is shown as the observed $17 \mathrm{MeV}$ for $34 M_{e}$ in the light boson (17eē ) [46] [47].

Quark has fractional charge $( \pm 1 / 3$ or $\pm 2 / 3)$, 3-color gluons (red, green, and blue) for $3 \mathrm{~g}^{*}$, and both the principal mass dimensions and axillary mass dimensions, so similar to Equation (31), $\mathrm{d}$ and $\mathrm{u}$ in the principal mass dimension involves $\mathrm{e} / 3$ or $2 \mathrm{e} / 3$ and $3 \mathrm{~g}^{*}$ as follows. 
principal mass dimensional oribital at $\mathrm{d}=6$

$$
\begin{aligned}
M_{\text {principal } q} & =\frac{1 \text { or } 2 M_{e}}{3}+\frac{3\left(3 M_{\mathrm{g}^{*}}\right)}{2} \\
& =\frac{1 \text { or } 2 M_{e}}{3}+\frac{3\left(3 M_{\mathrm{B}_{6}}\right)}{2} \\
& =\frac{1 \text { or } 2 M_{e}}{3}+\frac{9 M_{e}}{2 \alpha}
\end{aligned}
$$

For quarks in the auxiliary mass dimensions, 3-color basic gluons $\left(3 \mathrm{~g}^{\star}\right)$ become 3-color auxiliary basic gluons $\left(3 \mathrm{~g}^{*}{ }_{\mathrm{a} 7}\right)$ at $\mathrm{d}=7$. Based on Equation (29), auxiliary basic gluon is derived from muon as follows.

$$
M_{\mathrm{g}^{*}{ }^{*}}=M_{\mu 7} \alpha_{w}
$$

Similar to Equation (31), the masses of quarks in the auxiliary mass dimension are as follows.

$$
\begin{aligned}
& \text { auxiliary mass dimensional orbital at } \mathrm{d}=7 \\
& M_{\text {auxiliary } q_{7}}=\frac{3\left(3 M_{g^{*}{ }^{*} 7}\right)}{2} \sum_{a=1}^{n} a^{4}=\frac{9 M_{\mu 7} \alpha_{w}}{2} \sum_{a=1}^{n} a^{4}
\end{aligned}
$$

The quark mass formula at $\mathrm{d}=7$ is the combination of Equations (34) and (36) as follows.

$$
M_{q_{7}}=\frac{1 \text { or } 2 M_{e}}{3}+\frac{9 M_{e}}{2 \alpha}+\frac{9 M_{\mu 7} \alpha_{w}}{2} \sum_{a=1}^{n} a^{4}
$$

where $a=1,2,3,4$, and 5 for $\mathrm{u}_{7} / \mathrm{d}_{7}, \mathrm{~s}_{7}, \mathrm{c}_{7}, \mathrm{~b}_{7}$, and $\mathrm{t}_{7}$, respectively.

The quark mass at $a=5$ for the auxiliary mass dimension at $d=7$ is the maximum mass below the mass of $B_{7}$, so the next auxiliary mass dimension has to start from $\mathrm{B}_{7}$. There are $\mathrm{b}$ and $\mathrm{t}$ at $\mathrm{d}=8$, so it is necessary to have $\mu_{8}$ for the masses of $\mathrm{b}$ and t. Like $\mu_{7}$ in Equation (31), the mass of $\mu_{8}$ is as follows.

$$
\begin{aligned}
M_{\mu_{8}^{0}} & =2 M_{e}+3 M_{\mathrm{g}^{*}} / 2 \\
& =2 M_{e}+3 M_{\mathrm{B} 7} / 2 \\
& =2 M_{e}+3 M_{\mathrm{Z}^{0}} / 2 \\
& =136.78 \mathrm{GeV}
\end{aligned}
$$

Since at $\mathrm{d}=7$, there are 3-color basic gluons, at $\mathrm{d}=8,3$-color basic gluons are not needed, and only one basic gluon $\left(\mathrm{g}_{7}^{*}\right)$ at $\mathrm{d}=7$ is used. Similar to Equations (34) and (36), the quark mass formulas for the principal and auxiliary mass dimensions are as follows.

$$
\begin{aligned}
& \text { principal mass dimensional orbital at } \mathrm{d}=7 \\
& M_{\text {principal quark }}=3 M_{g^{*}} / 2=3 M_{B_{7}} / 2=3 M_{Z} / 2 \\
& \text { auxiliary mass dimensional orbital at } \mathrm{d}=8 \\
& M_{\text {auxiliary quark }}=\frac{3\left(M_{g^{*}}\right)}{2} \sum_{a^{\prime}=1}^{n^{\prime}} a^{\prime 4}=\frac{3 \mu_{8}^{0} \alpha}{2} \sum_{a^{\prime}=1}^{n^{\prime}} a^{\prime 4}
\end{aligned}
$$

The quark mass formula at $\mathrm{d}=8$ is the combination of Equations (37) and (39) as follows. 


$$
M_{q 8}=\frac{3 M_{Z}}{2}+\frac{3 M_{\mu_{8}^{0}} \alpha}{2} \sum_{a^{\prime}=1}^{n^{\prime}} a^{\prime 4}
$$

where $a^{\prime}=1$ and 2 for $b_{8}$ and $t_{8}$, respectively.

Combining Equations (37) and (41), the quark mass formula is as follows.

$$
M_{\text {quark }}=\frac{1 \text { or } 2 M_{e}}{3}+\frac{9 M_{e}}{2 \alpha}+\frac{9 M_{\mu_{7}} \alpha_{w}}{2} \sum_{a=1}^{n} a^{4}+\frac{3 M_{Z}}{2}+\frac{3 M_{\mu_{8}^{0}} \alpha}{2} \sum_{a^{\prime}=1}^{n^{\prime}} a^{\prime 4}
$$

where $a=1,2,3,4$, and 5 for $\mathrm{d} / \mathrm{u}$. s, c, b, and t, respectively, and $\mathrm{a}^{\prime}=1$ and 2 for $\mathrm{b}$ and $\mathrm{t}$ respectively. The calculated masses for $\mathrm{d}, \mathrm{u}, \mathrm{s}, \mathrm{c}, \mathrm{b}$, and $\mathrm{t}$ are $328.4 \mathrm{MeV}$, 328.6 MeV, $539 \mathrm{MeV}, 1605.3 \mathrm{MeV}, 4974.6 \mathrm{MeV}$, and $175.4 \mathrm{GeV}$, respectively. In the standard model, there are three generations of leptons. Extra-muon $\mu_{8}$ is outside of the three generations of leptons in the standard model, so $\mu_{8}$ is absent as shown in Table 2 . As shown in Table 2 , to be symmetrical to the absent $\mu_{8}, \mathrm{~b}_{8}$ quark is also absent. The calculated mass of top quark is $175.4 \mathrm{GeV}$ in good agreement with the observed $172.4 \mathrm{GeV}$ [44]. The calculated masses are comparable to the quark masses proposed by De Rujula, Georgi, and Glashow [48], Griffiths [49], and El Naschie [50].

The periodic table of elementary particles calculates accurately the particle masses of all leptons, quarks, gauge bosons, the Higgs boson, and the cosmic rays by using only five known constants: the number (seven) of the extra spatial dimensions in the observed four-dimensional spacetime from the eleven-dimensional membrane, the mass of electron, the masses of $\mathrm{Z}$ and $\mathrm{W}$ bosons, and the fine structure constant [21] [22] [51]. The calculated masses are in excellent agreements with the observed masses. For example, the calculated masses of muon, top quark, and the Higgs boson are $105.55 \mathrm{MeV}, 175.4 \mathrm{GeV}$, and 126 $\mathrm{GeV}$, respectively, in excellent agreements with the observed $105.65 \mathrm{MeV}, 172.4$ $\mathrm{GeV}$, and $126 \mathrm{GeV}$, respectively. The calculated masses of hadrons based on the periodic table of elementary particles are in excellent agreement with the observed masses of hadrons [22]. For examples, the calculated masses of proton, neutron, pion $\left(\pi^{ \pm}\right)$, and pion $\left(\pi^{0}\right)$ are $938.261,939.425,139.540$, and 134.982 $\mathrm{MeV}$ in excellent agreement with the observed 938.272, 939.565, 139.570, and $134.977 \mathrm{MeV}$, respectively with $0.0006 \%, 0.01 \%, 0.02 \%$, and $0.004 \%$, respectively for the difference between the calculated and observed mass.

\subsection{The Three Branes}

The other three 10D string branes (negative energy weak-gravity brane, positive energy strong-gravity brane, and negative energy strong-gravity brane) did not absorb detachment space, but to synchronize the expansion of the positive-energy string brane (home universe), the other three 10D string branes undergo the oscillation between $10 \mathrm{D}$ and $4 \mathrm{D}$.

\subsubsection{The Hidden Three Branes from 10D to 5D}

The three branes undergo the transformation from $10 \mathrm{D}$ to $5 \mathrm{D}$. 


$$
\begin{aligned}
& 10 \mathrm{D} 4 \mathrm{~d} \rightarrow \text { 9D5d } \rightarrow \text { 9D4d } \rightarrow \text { 8D5d } \rightarrow \text { 8D4d } \rightarrow \text { 7D } 5 d \\
& \rightarrow \text { 7D4d } \rightarrow \text { 6D5d } \rightarrow \text { 6D4d } \rightarrow \text { 5D5d } \rightarrow \text { 5D4d }
\end{aligned}
$$

From Equation (21), under the VSLD transformation and the VSD transformation, the three branes expand through the increasing rest mass and the translation-fractionalization from $10 \mathrm{D} 4 \mathrm{~d}$ to $5 \mathrm{D} 4 \mathrm{~d}$. To the $4 \mathrm{D}$ home universe, the three branes from 10D to 5D are hidden, because as Equation (13), particles with different space-time dimensions and different speeds of light are transparent and oblivious to one another to avoid the violation of causality due to differences in the speed of light. During this time, the home 4D universe expands normally.

\subsubsection{The Three Branes as Dark Energy}

When all three branes and the home universe become $4 \mathrm{D}$, the three branes become dark energy as a part of the home universe.

$$
4 \mathrm{D} 5 \mathrm{~d} \rightarrow \text { 4D4d }
$$

The result is the accelerating expansion. Since the three branes have no detachment space to produce kinetic energy, dark energy is inert as the inert cosmological constant. According to the theoretical calculation based on the algebras cosmology, dark energy started in 4.47 billion years ago [19] in agreement with the observed $4.71 \pm 0.98$ billion years ago [52]. The maximum dark energy is $75 \%$ for the three out of the four regions as the spacetime of three branes becomes completely $4 \mathrm{D}$.

\subsubsection{The Three Hidden Branes from 5D to 10D}

The three branes from 5D to 10D again become the hidden branes.

$$
\begin{aligned}
& 5 \mathrm{D} 4 \mathrm{~d} \rightarrow 5 \mathrm{D} 5 \mathrm{~d} \rightarrow 6 \mathrm{D} 4 \mathrm{~d} \rightarrow 6 \mathrm{D} 5 \mathrm{~d} \rightarrow 7 \mathrm{D} 4 \mathrm{~d} \rightarrow 7 \mathrm{D} 5 \mathrm{~d} \\
& \rightarrow \text { D4 } \rightarrow \text { d } \rightarrow \text { 5 d } \rightarrow \text { 9D4d } \rightarrow \text { 9D5d } \rightarrow \text { 10D4d }
\end{aligned}
$$

They contract by the decreasing rest mass and the translation-condensation. The home universe contracts through gravity. Through symmetry, all four universes contract synchronically and equally.

\subsubsection{The Three 10D Branes and the Original 4D Home Universe}

Eventually, the three branes return to the original $10 \mathrm{D}$ three branes. The $4 \mathrm{D}$ home universe reaches cosmic deflation to lose all detachment space to become 10D4d. The four universes return to the step 3.

In the home universe

4D home universe $\stackrel{\text { cosmic deflation }}{\longrightarrow}$ positive energy 10D4d weak gravity brane In the three branes

9D5d branes $\longrightarrow$ the 10D4d branes

The four branes can undergo another cycle of the home universe and the three branes or can reverse to the 11D membrane-antimembrane dual universes, and ultimately, to the zero-energy inter-universal void as shown in Figure 6.

\section{Summary}

In summary, the cyclic universe cosmology involves the split of the membrane 
11D (11 dimensional) spacetime into the 1D eleventh dimension orbifold interval space to form gravity, the $6 \mathrm{D}$ discrete interior rishon space (TTT-VVV for positron-neutrino or TTV-TVV for u-d quarks) to form the Standard Model, the 3D Higgs space (attachment space to attach matter or detachment space to detach matter) to form the Higgs or reverse Higgs field, and 1D Einstein time to be shared by all spaces. The $1 \mathrm{D}$ eleventh dimension interval space was the space between the weak-gravity string brane and the strong-gravity string brane which are the progenitors of the home universe and dark energy, respectively.

The 6D discrete interior rishon space consists of the TTT-VVV or TTV-TVV as the discrete trivalent twisted strand braids for the spaces of positron-neutrino or u-d quarks, respectively, in the Rishon Space Model with $1 / 3$ charge $\mathrm{T}$ and neutral V. The trivalent discrete rishon space is the interior space to determine the properties (flavor, charge, isospin, and color) of leptons and quarks, while the trivalent connected space is the exterior space to determine the momenta and positions of leptons and quarks.

The 3D Higgs space consists of attachment space as the space precursor of the transitional Higgs field and detachment space as the space precursor of the transitional reverse Higgs field. Attachment space that attaches matter to the space related to rest mass, detachment space that detaches matter from the space relates to kinetic energy. The combination of $n$ units of attachment space (denoted as 1 ) and $n$ units of detachment space (denoted as 0 ) produces binary partition space, $(1)_{n}(0)_{n}$, as the space of wave-particle duality, binary miscible space, $(1+0)_{n}$, as the space of relativity, and binary lattice space, $(1,0)_{n}$, as the space of virtual gauge boson in quantum field theory. A particle with the rishon space is bound to the different Higgs spaces under different conditions. The Higgs space is the interior space to determine the properties (attachment and detachment to matter) of elementary particles, while the exterior space determines the momenta and positions of elementary particles.

Between 4D spacetime and 10D spacetime, dimension number decreases with decreasing speed of light, decreasing vacuum energy, and increasing rest mass. The $4 \mathrm{D}$ and the $10 \mathrm{D}$ have zero and the highest vacuum energies, respectively. Each spacetime dimension $\mathrm{D}$ associates with mass dimension $\mathrm{d}$ where $\mathrm{D}+\mathrm{d}=$ 14.

This paper posits that the origin of the split is from the multiverse cosmology. The multiverse consists of the $4 \mathrm{D}$ zero-energy inter-universal void with detachment space and the multiple $11 \mathrm{D}$ positive-energy membrane and negative-energy anti-membrane dual universes with $11 \mathrm{D}$ attachment space. The total energy of a dual 11D universe is zero. Detachment space in the inter-universal void prevents the collision of the $11 \mathrm{D}$ dual universes, while attachment space in a dual universe allows the existence of mass-energy in the space of the dual universe. A dual universe with zero total energy can emerge from the zero-energy inter-universe.

The cyclic universe cosmology (Figure 6) in the multiverse starts with the zero-energy inter-universal void and the positive-energy membrane and nega- 
tive-energy antimembrane 11D dual universe which is split into four equal 10D string branes, including the $10 \mathrm{D}$ positive-energy weak-gravity brane with matter, negative-energy strong-gravity brane, negative-energy weak-gravity brane with antimatter, and positive-energy strong-gravity brane in the $11 \mathrm{D}$ bulk. The $1 \mathrm{D}$ eleventh dimension interval space is between the strong and the weak-gravity branes as in Randall-Sundrum model.

To form the home universe where we inhabit, the 10D positive-energy weak-gravity brane with attachment space absorbed the zero-energy $4 \mathrm{D}$ inter-universal void with detachment space, resulting in the combination of rest mass from attachment space and kinetic energy from detachment space, the formation of the $4 \mathrm{D}$ spacetime universe by transforming $6 \mathrm{D}$ connected exterior space into 6D discrete interior rishon space to form the extended Standard Model, and cosmic inflation from high vacuum energy (10D) to zero vacuum energy (4D). The other three branes did not absorb the inter-universal void, resulting in the oscillating dimension branes between 10D and 4D stepwise without kinetic energy. The three branes are hidden when $\mathrm{D}>4$, and they are dark energy when $\mathrm{D}=4$. Afterward, when $\mathrm{D}>4$ again for the three branes, the home universe and the three branes start to contract, and eventually return to the original four 10D string branes and then to the original 11D membrane dual universe for the cyclic universe cosmology as in Figure 6.

Consequently, the emergences of gravity, the Standard Model, and dark energy as the three different phenomena take three steps. The Step 1 to form gravity involves the emergence of the $1 \mathrm{D}$ interval space to form gravity derived from the split of the 11D membrane universe into two branes with 1D interval space in between the two branes. The Step 2 to form the Standard Model involves the 6D rishon space to form the Standard Model derived from the addition of detachment space to the $10 \mathrm{D}$ weak-gravity brane to form the home universe consisting of the combination of attachment space and detachment space for the Higgs mechanism and the 4D spacetime with 6D TTTVVV or TTVTVV for the origin of elementary particles. The Step 3 to form dark energy involves the $4 \mathrm{D}$ strong-gravity brane to form dark energy derived from the dimensional oscillation between 10D and 4D. The 3-step cosmic evolution from the beginning of the cyclic universe to the present is as Figure 9.

All elementary particles for baryonic matter (leptons, quarks, gauge bosons, gravity, and the Higgs boson) and dark matter (sterile neutrinos) can be placed in the periodic table of elementary particles based on the two sets of the seven mass dimensions. The periodic table of elementary particles calculates accurately the particle masses of all leptons, quarks, gauge bosons, the Higgs boson, and the cosmic rays by using only five known constants: the number (seven) of the extra spatial dimensions in the observed four-dimensional spacetime from the eleven-dimensional membrane, the mass of electron, the masses of $\mathrm{Z}$ and $\mathrm{W}$ bosons, and the fine structure constant. The calculated masses are in excellent agreements with the observed masses. For examples, the calculated masses of muon, top quark, and the Higgs boson are $105.55 \mathrm{MeV}, 175.4 \mathrm{GeV}$, and 126 


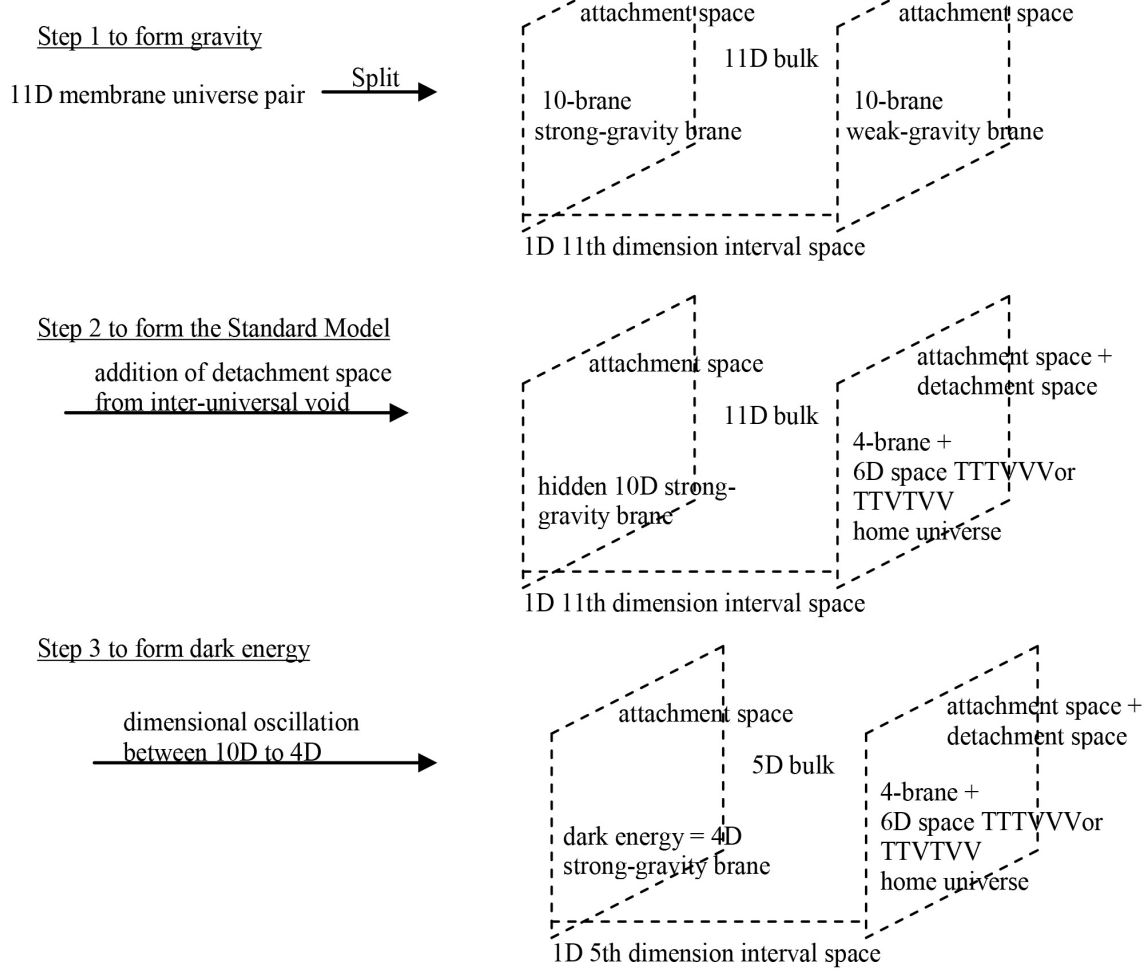

Figure 9. The 3-step cosmic evolution from the beginning to the present. Step1 to form gravity: split the 11D membrane universe into two branes with $1 \mathrm{D}$ interval space in between the two branes. Step 2 to form the Standard Model: the addition of detachment space to the $10 \mathrm{D}$ weak-gravity brane to form the home universe with the combination of attachment space and detachment space and the 6D rishon space. Step 3 to form dark energy: the conversion of the strong-gravity brane to the $4 \mathrm{D}$ dark energy by the dimensional oscillation between $10 \mathrm{D}$ and $4 \mathrm{D}$ from the strong-gravity brane.

$\mathrm{GeV}$, respectively, in excellent agreements with the observed $105.65 \mathrm{MeV}, 172.4$ $\mathrm{GeV}$, and $126 \mathrm{GeV}$, respectively. The calculated masses of hadrons based on the periodic table of elementary particles are in excellent agreement with the observed masses of hadrons. For examples, the calculated masses of proton, neutron, pion $\left(\pi^{ \pm}\right)$, and pion $\left(\pi^{0}\right)$ are $938.261,939.425,139.540$, and $134.982 \mathrm{MeV}$ in excellent agreement with the observed 938.272, 939.565, 139.570, and 134.977 $\mathrm{MeV}$, respectively with $0.0006 \%, 0.01 \%, 0.02 \%$, and $0.004 \%$, respectively for the difference between the calculated and observed mass. In conclusion, the split 11D spacetime, and cyclic universe cosmology, and the periodic table of elementary particles provide the matter-antimatter imbalance and the accurate calculated masses for leptons, quarks, hadrons, gauge bosons, the Higgs boson, gravity, dark matter, and dark energy

\section{Conflicts of Interest}

The author declares no conflicts of interest regarding the publication of this paper. 


\section{References}

[1] Green, M., Schwarz, J. and Witten, E. (2012) Superstring Theory. Vol. 1: Introduction. Cambridge University Press, Cambridge.

[2] Woit, P. (2006) Not Even Wrong: The Failure of String Theory and the Search for Unity in Physical Law. Basic Books, New York.

[3] Randall, L. and Sundrum, R. (1999) Physical Review Letters, 83, 4690-4693. https://doi.org/10.1103/PhysRevLett.83.4690

[4] Randall, L. (2005) Warped Passages: Unraveling the Mysteries of the Universe's Hidden Dimensions. Harper Collins, New York.

[5] Randall, L. and Sundrum, R. (1999) Physical Review Letters, 83, 3370-3373. https://doi.org/10.1103/PhysRevLett.83.3370

[6] Pati, J. and Salam, A. (1974) Physical Review D, 10, 275-289. https://doi.org/10.1103/PhysRevD.10.275

[7] Dugne, J., Fredriksson, S. and Hansson, J. (2002) Europhysics Letters, 57, 188-194. https://doi.org/10.1209/epl/i2002-00337-8

[8] Harari, H. (1979) Physics Letters B, 86, 83-86. https://doi.org/10.1016/0370-2693(79)90626-9

[9] Shupe, M. (1979) Physics Letters B, 86, 87-92. https://doi.org/10.1016/0370-2693(79)90627-0

[10] Harari, H. and Seiberg, N. (1982) Nuclear Physics B, 204, 141-167. https://doi.org/10.1016/0550-3213(82)90426-6

[11] Brodsky, S. and Drell, S. (1980) Physical Review D, 22, 2236-2243. https://doi.org/10.1103/PhysRevD.22.2236

[12] Bilson-Thompson, S. (2005) A Topological Model of Composite Preons.

[13] Bilson-Thompson, S., Hackett, J., Kauffman, L. and Wan, Y. (2012) Symmetry, Integrability and Geometry: Methods and Applications, 8, 14. https://doi.org/10.3842/SIGMA.2012.014

[14] Barut, A. and Chung, D. (1983) Lettere al Nuovo Cimento, 38, 225-226. https://doi.org/10.1007/BF02814632

[15] Weinberg, S. (1989) Review Modern Physics, 61, 1-23. https://doi.org/10.1103/RevModPhys.61.1

[16] Chung, D. (2016) Journal of Modern Physics, 7, 1210-1227. https://doi.org/10.4236/jmp.2016.710110

[17] Hansson, J. (2015) International Journal of Modern Physics and Applications, 1, 12-16.

[18] Jammer, M. (2009) Concepts of Mass in Contemporary Physics and Philosophy. Princeton University Press, Princeton. https://doi.org/10.1515/9781400823789

[19] Chung, D. and Krasnoholovets, V. (2013) Journal of Modern Physics, 4, 77-84. https://doi.org/10.4236/jmp.2013.47A1009

[20] Chung, D. (2018) Journal of Modern Physics, 9, 2257-2273. https://doi.org/10.4236/jmp.2018.913142

[21] Chung, D. (2018) Journal of Modern Physics, 9, 2308-2319. https://doi.org/10.4236/jmp.2018.913146

[22] Chung, D. (2018) Journal of Modern Physics, 9, 2638-2656. https://doi.org/10.4236/jmp.2018.914164 
[23] Gorham, P., et al. (2016) Physical Review Letters, 117, Article ID: 071101.

[24] Gorham, P., et al. (2018) Physical Review Letters, 121, Article ID: 161102.

[25] Gorham, P., et al. (2009) Astroparticle Physics, 32, 10-41.

[26] Penrose, R. (2000) Wavefunction Collapse as a Real Gravitational Effect. In: Fokas, A., Grigoryan, A., Kibble, T. and Zegarlinski, B., Eds., Mathematical Physics, Imperial College, London, 266-282. https://doi.org/10.1142/9781848160224_0013

[27] Moffat, J. (1993) International Journal of Modern Physics D, 2, 351-359. https://doi.org/10.1142/S0218271893000246

[28] Albrecht, A. and Magueijo (1999) Physics Review D, 59, Article ID: 043516. https://doi.org/10.1103/PhysRevD.59.043516

[29] Barrow, J. (2003) Physics Letters B, 564, 1-7. https://doi.org/10.1016/S0370-2693(03)00573-2

[30] Bounias, M. and Krasnoholovets, V. (2003) The International Journal of Systems and Cybernetics, 32, 1005-1020. https://doi.org/10.1108/03684920310483144

[31] Jarosik, N., et al. (2011) The Astrophysical Journal Supplement Series, 192, 14. https://doi.org/10.1088/0067-0049/192/2/14

[32] Fujita, J. and Miyazawa, H. (1957) Progress of Theoretical Physics, 17, 360. https://doi.org/10.1143/PTP.17.360

[33] Chung, D. (2016) Journal of Modern Physics, 7, 1150-1159. https://doi.org/10.4236/jmp.2016.710104

[34] Tsui, D., Stormer, H. and Gossard, A. (1982) Physical Review Letters, 48, 1559-1562. https://doi.org/10.1103/PhysRevLett.48.1559

[35] Stormer, H. (1999) Reviews of Modern Physics, 71, 875-889. https://doi.org/10.1103/RevModPhys.71.875

[36] Laughlin, R. (1983) Physical Review Letters, 50, 1395-1398. https://doi.org/10.1103/PhysRevLett.50.1395

[37] Loureiro, A., et al. (2019) Physical Review Letters, 123, Article ID: 081301. https://doi.org/10.1103/PhysRevLett.123.081301

[38] Chung, D. (2015) Global Journal of Science Frontier Research A, 15, 119-125.

[39] Moffat, J. (2006) Journal of Cosmology and Astroparticle Physics, 3, 4. https://doi.org/10.1088/1475-7516/2006/03/004

[40] Moffat, J. (2008) Reinventing Gravity. HarperCollins, New York.

[41] Chung, D. (2014) International Journal of Astronomy and Astrophysics, 4, 374-383. https://doi.org/10.4236/ijaa.2014.42032

[42] Kamilla, R., Wu, X. and, Jain, J. (1996) Physics Review Letters, 76, 1332. https://doi.org/10.1103/PhysRevLett.76.1332

[43] Jain, J. (2007) Composite Fermions. Cambridge University Press, New York.

[44] Particle Data Group (2018) Physical Review D, 98, Article ID: 030001. https://doi.org/10.1103/PhysRevD.98.030001

[45] Barut, A. (1979) Physical Review Letters, 42, 1251. https://doi.org/10.1103/PhysRevLett.42.1251

[46] Krasznahorkay, A., et al. (2016) Physical Review Letters, 116, Article ID: 042501. https://doi.org/10.1103/PhysRevLett.116.042501

[47] Feng, J., et al. (2016) Physical Review Letters, 117, Article ID: 071803. https://doi.org/10.1103/PhysRevLett.117.071803 
[48] De Rujula, A., Georgi, H. and Glashow, S. (1975) Physics Review D, 12, 147-162. https://doi.org/10.1103/PhysRevD.12.147

[49] Griffiths, D. (2008) Introduction to Elementary Particles. Wiley-VCH, Weinheim.

[50] El Naschie, M. (2002) Chaos, Solitons and Fractals, 14, 369-376. https://doi.org/10.1016/S0960-0779(02)00022-X

[51] Chung, D. (2016) Journal of Modern Physics, 7, 1591-1606. https://doi.org/10.4236/jmp.2016.712144

[52] Riess, A.G., et al. (2004) Astrophysical Journal, 607, 665-687. https://doi.org/10.1086/383612 